Draft VERSION July 18, 2018

Preprint typeset using IATEX style emulateapj v. 11/12/01

\title{
A SEARCH FOR MID-INFRARED EMISSION FROM HOT MOLECULAR CORE CANDIDATES
}

\author{
J. M. De Buizer ${ }^{1,2,3}$ \\ Gemini Observatory, Casilla 603, La Serena, Chile \\ jdebuizer@gemini.edu \\ J. T. Radomski, C. M. Telesco ${ }^{2}$, And R. K. PiñA ${ }^{2,4}$ \\ Department of Astronomy, University of Florida, Gainesville, FL 32611, USA \\ Draft version July 18, 2018
}

\begin{abstract}
We present here mid-infrared images of seven sites of water maser emission thought to be associated with the hot molecular core (HMC) phase of massive star formation. Observations were obtained at the NASA InfraRed Telescope Facility 3-m, the Gemini 8-m, and Keck II 10-m telescopes. We have detected mid-infrared sources at the locations of two HMC candidates, G11.94-0.62 and G45.07-0.13. We observed G19.61-0.23 and G34.26+0.15, each of which have HMCs previously detected in the mid-infrared. We did not detect mid-infrared emission from either HMC source, and we place new upper limits on the midinfrared flux densities for these HMCs that are much lower than their previously reported flux densities. We were able to obtain extremely accurate astrometry for our mid-infrared images of G9.62+0.19, and conclude that the mid-infrared emission thought to be coming from the HMC in this field is in fact coming from a different source altogether.
\end{abstract}

Subject headings: circumstellar matter - infrared: ISM - stars: early type - stars: formation - masers

\section{INTRODUCTION}

Hot molecular cores (HMCs) are believed to represent an extremely early stage of massive stellar birth. An HMC consists of a massive protostar surrounded by a thick envelope of accreting dust and gas. Though there are very few observations of these objects to date, we are beginning to piece together an empirically derived list of the basic observational characteristics of HMCs: 1) they are compact sources seen in radio-wavelength ammonia (or molecular line) images but have little or no radio continuum emission; 2) they lie in massive star forming regions near ultracompact HiI (UC HiI) regions; 3) they are too young and/or embedded to be seen in the optical or near infrared; and 4) they are often coincident with water maser emission. Only a small number of sources exist that have had such a holistic set of observations performed.

The work by Cesaroni et al. (1994) remains the most comprehensive work on the subject, even though their work involved radio-wavelength ammonia line imaging towards only four fields of massive star formation. In all four cases they found compact ( $\sim 0.1 \mathrm{pc})$ ammonia clumps offset from, but near to, UC Hir regions. In all four cases the water masers were directly coincident with the ammonia emission rather than the radio continuum emission of the UC HiI region. Cesaroni et al. (1994) observed in the high-excitation $(4,4)$ line of $\mathrm{NH}_{3}$, which traces gas with kinetic temperatures of 50-200 K, and densities approximating $\mathrm{n}_{H_{2}} \sim 10^{7} \mathrm{~cm}^{-3}\left(\mathrm{n}_{N H_{3}} / \mathrm{n}_{H_{2}}>10^{-6}\right)$ within these HMCs.

Cesaroni et al. (1994) also argue that gas and dust would be well mixed in the HMCs and that there would be a high rate of collisions between the dust and gas. When densities in excess of $\mathrm{n}_{\mathrm{H}_{2}} \gtrsim 10^{5} \mathrm{~cm}^{-3}$ exist, temperature equilibrium between gas and dust is established (Krugel and Walmsley 1984). Given the high observed densities of HMCs, it can be concluded that the gas kinetic temperature is a fair approximation to the dust temperature. The observed gas temperatures for HMCs is typically between 50 and $200 \mathrm{~K}$ (Cesaroni et al. 1994). Therefore one should be able to detect new HMCs via their mid-infrared emission, at least for the warmest sources.

Recently there was a detection of mid-infrared emission from the HMC of G29.96-0.02 (De Buizer et al. 2002), a source that was first imaged by Cesaroni et al. (1994) in $\mathrm{NH}_{3}$ emission. This source is also in the survey of Hofner \& Churchwell (1996), who studied the relationship between the $2 \mathrm{~cm}$ radio continuum emission of UC HII regions and the location of the water masers on the field. They found that G29.96-0.02, like several other sources in their survey, did not have water masers associated with the observed UC HII regions. Instead, the water masers tend to be in groups that lie offset from the radio continuum emission. The hypothesis is that the water masers are excited by embedded massive stellar sources within the HMCs. It is pointed out by Hofner \& Churchwell (1996) that several of the UC Hir regions in their survey are cometary-shaped, like G29.96-0.02, and have their water masers located in front of the cometary arc of radio continuum emission.

Another source in common between the Cesaroni et al. (1994) survey and Hofner \& Churchwell (1996) survey is G9.62+0.19. Hofner \& Churchwell (1996) showed that both sources display radio continuum regions with water masers offset, and Cesaroni et al. (1994) showed that both have HMCs at the water maser locations as seen in $\mathrm{NH}_{3}$.

\footnotetext{
${ }^{1}$ Visiting Astronomer, Gemini North Observatory and NASA Infrared Telescope Facility.

${ }^{2}$ Visiting Astronomer, W. M. Keck Observatory.

${ }^{3}$ On leave from Cerro Tololo Inter-American Observatory, Casilla 603, La Serena, Chile

${ }^{4}$ Present address: Photon Research Associates, Inc., 5720 Oberlin Drive, San Diego, CA 92121, USA
} 
In order to extend our knowledge of the earliest stages of massive star formation, and understand more about the nature of HMCs, we have undertaken mid-infrared observations of several fields from the survey of Hofner \& Churchwell (1996) in an attempt to find more midinfrared-bright HMCs. We concentrate on the fields that have UC Hir regions with water masers well offset. Some of these fields are the sites of already detected HMCs at the water maser locations (e.g. imaged in molecular lines of $\mathrm{NH}_{3}$, or already imaged in the mid-infrared). For the other fields, observations of molecules found in HMCs but not UC HiI regions can be used to deduce the physical presence of HMCs. Fields that do not have directly detected HMCs were checked for spectroscopic observations of molecular species known to be tracers of the densest and warmest material. In particular, methyl cyanide $\left(\mathrm{CH}_{3} \mathrm{CN}\right)$ and high excitation lines of $\mathrm{NH}_{3}$ appear to be confined to those HMCs that are luminous at far-infrared and submillimeter wavelengths. From the list of Hofner \& Churchwell (1996) we have therefore isolated seven HMCs and/or HMC candidates for detailed imaging in the mid-infrared. While all sources were observed on the IRTF $3-\mathrm{m}$, others needed high spatial resolution or deep mid-infrared imaging as follow-up. These sources were observed on the Gemini 8-m and Keck II 10-m telescopes.

\section{OBSERVATIONS}

Exploratory observations were first performed using the University of Florida mid-infrared camera and spectrometer, OSCIR, in 1997 September at the 3-meter NASA InfraRed Telescope Facility (IRTF). OSCIR employs a Boeing/Rockwell ${ }^{5} 128$ x 128 Si:As BIB (blocked impurity band) detector array, which is optimized for wavelength coverage between 8 and $25 \mu \mathrm{m}$. The field of view of the array is $29^{\prime \prime} \times 29^{\prime \prime}$, for a scale of $0.223^{\prime \prime} /$ pixel. Observations were centered on the $\mathrm{H}_{2} \mathrm{O}$ maser coordinates given by Hofner \& Churchwell (1996), with 60 sec on-source exposure times taken through a broad-band $N$ filter $\left(\lambda_{0}=\right.$ $10.46 \mu \mathrm{m}, \Delta \lambda=5.1 \mu \mathrm{m}$ ) and the $I H W 18$ (International Halley Watch, $\lambda_{0}=18.06 \mu \mathrm{m}, \Delta \lambda=1.7 \mu \mathrm{m}$ ) filter with a $30^{\prime \prime} \mathrm{N}-\mathrm{S}$ chop throw. Unfortunately, cirrus clouds terminated the survey after only three sites were imaged.

The full survey was performed with deeper imaging in 2002 June, again at the IRTF, but this time using the Jet Propulsion Laboratory mid-infrared camera, MIRLIN. This instrument employs a Boeing ${ }^{5}$ HF-16 128 x 128 Si:As BIB detector array. The pixel-scale is $0.475^{\prime \prime} /$ pixel, for a field of view of $61^{\prime \prime} \times 61^{\prime \prime}$. Observations were taken through the $N_{4}\left(\lambda_{0}=11.70 \mu \mathrm{m}, \Delta \lambda=1.11 \mu \mathrm{m}\right)$ and $Q 3$ $\left(\lambda_{0}=20.81 \mu \mathrm{m}, \Delta \lambda=1.65 \mu \mathrm{m}\right)$ filters with on-source exposures times of 184 and $192 \mathrm{sec}$, respectively. All observations were taken at airmasses $<1.5$ under clear skies with low relative humidity $(<25 \%)$ with a chop throw of $61^{\prime \prime}$ N-S. Sky and telescope subtraction were removed using the standard chop-nod technique, and the chop throw was large enough that there was no contamination from sources in the reference chop beam. The primary standard star used throughout the observations was $\gamma$ Aql, for which the flux densities were taken to be $61.1 \mathrm{Jy}$ at $11 \mu \mathrm{m}$ and $19.5 \mathrm{Jy}$ at $20 \mu \mathrm{m}$.

Follow-up high-resolution observations were performed on two separate occasions. First, an observation of G9.62+0.19 was performed in 1999 April at the W.M. Keck II 10-m telescope. This observation was made using the OSCIR camera, a visiting instrument at the time. A single exposure was made of G9.62+0.19, with an onsource integration time of $240 \mathrm{sec}$ through the $I H W 18$ filter. At Keck, OSCIR has a field of view of $8^{\prime \prime} \times 8^{\prime \prime}$, for a scale of $0.0616^{\prime \prime} /$ pixel. On the second occasion in 2001 May, observations were made at the Gemini North 8-m telescope of G19.61-0.23 and G34.26+0.15, again using OSCIR. One image was taken of G19.61-0.23 through the $I H W 18$ filter for $180 \mathrm{sec}$. Images of G34.26+0.15 were taken through both the $N$ and $I H W 18$ filters with 120 sec exposure times. At Gemini North, OSCIR has a field of view of $11^{\prime \prime} \times 11^{\prime \prime}$, for a scale of $0.084^{\prime \prime} /$ pixel. Like the IRTF observations, all of these high-resolution observations were taken at airmasses $<1.5$ under clear skies, and sky and telescope subtraction were removed using the standard chop-nod technique. Chop throws were $10^{\prime \prime} \mathrm{N}-\mathrm{S}$ at Keck and $15^{\prime \prime} \mathrm{E}-\mathrm{W}$ at Gemini. The standard star used at Keck and Gemini North was $\alpha$ Lyr, with an assumed flux density of $36.4 \mathrm{Jy}$ at $10.5 \mu \mathrm{m}$ and $12.0 \mathrm{Jy}$ at $18.1 \mu \mathrm{m}$.

Table 1 lists the seven target fields in this survey. The table gives for each target field the coordinates of the water maser group number from Hofner \& Churchwell (1996) that is either associated with a known HMC, or is the likely location of the HMC candidate. The water maser positions of Hofner \& Churchwell (1996) were obtained with a connected element interferometer and have absolute positional uncertainties less than $0 . .5$. The mid-infrared absolute astrometry was obtained at IRTF by offsetting the telescope to the maser reference coordinates from nearby reference stars (typically less than $10^{\prime}$ away) whose coordinates were obtained from the Hipparcos Catalog. The absolute pointing uncertainty of the telescope was estimated to be better than $1^{\prime \prime} .5$ in repeated trials, determined by offsetting between nearby Hipparcos reference stars. This astrometric accuracy is consistent with the pointing uncertainties previously achieved at IRTF during similar observations by Campbell et al. (2000). We therefore conclude that the relative uncertainty of the astrometry between the maser reference features and our pointing is also better than $1^{\prime \prime} .5$, in general. However, for each source detected we could confirm or increase this astrometric accuracy by comparing the mid-infrared field to the field imaged at other wavelengths. This process is discussed on a case-by-case basis for each source in $\S 3$.

The standard star images were used as point-spread function (PSF) stars for the IRTF observations. The measured resolution of the images was taken to be the average of the size of the full width at half maximum (FWHM) of the PSF stars imaged throughout the course of the observations. At IRTF the PSF observations yield an image resolution of $1^{\prime \prime} .2$ at $10.5 \mu \mathrm{m}$ and $1^{\prime \prime} .6$ at $18.1 \mu \mathrm{m}$ on the night using OSCIR, and $1^{\prime \prime} .3$ at $11.7 \mu \mathrm{m}$ and $1^{\prime \prime} .7$ at 20.8 $\mu \mathrm{m}$ on two nights when MIRLIN was used. At Gemini and Keck, PSF stars were imaged near the location of each of the target fields imaged. The Gemini observations have a average measured resolution of 0.48 at $10.5 \mu \mathrm{m}$ and 0.63 at $18.1 \mu \mathrm{m}$, and PSF observations at Keck yielded a resolution of $0^{\prime \prime} .33$ at $10.5 \mu \mathrm{m}$ and 0.41 at $18.1 \mu \mathrm{m}$.

${ }^{5}$ Both Boeing and Rockwell are now subsidiaries of DRS Technologies. 
Table 1 lists the observed flux densities of the HMC candidates at four wavelengths. The 10.5 and $18.1 \mu \mathrm{m}$ flux densities correspond to the OSCIR data from various telescopes (which are annotated), and the 11.7 and $20.8 \mu \mathrm{m}$ flux densities are from MIRLIN from IRTF. This paper concentrates discussion only on the HMC candidates on each field and reserves discussion of all other mid-infrared sources for future articles. However, since most of these fields have not been imaged in the mid-infrared before, Table 2 lists the flux densities for all mid-infrared sources found on all fields. Sources are designated by numbers which are incremented by increasing right ascension on the field ${ }^{6}$. The position of these sources are given as offsets in arcseconds from the position of the HMC on the field as given in Table 1. The flux densities listed in both tables are given with no quoted error. The largest sources of error in these values is the atmospheric variability the night of the observations and the uncertainty in the mid-infrared standard star flux density in each filter. The largest observed sky variability for any night of observations was approximately $10 \%$ at 10.5 and $11.7 \mu \mathrm{m}$ and $15 \%$ at 18.1 and $20.8 \mu \mathrm{m}$. These values were obtained from the variability of the measured detector counts for a given standard star throughout the night. We therefore assign the derived flux densities in this paper the above stated errors as conservative estimates of the actual photometric errors for all observations.

\section{RESULTS FOR INDIVIDUAL SOURCES}

\section{1. $G 9.62+0.19$}

G9.62+0.19 and its surrounding environment have been well studied at a variety of wavelengths (see Testi et al. 2000 and references therein). This complex region contains a wealth of high mass sources of different evolutionary states, from the HMC phase to well-developed His regions. A study of the centimeter continuum emission from this region by Garay et al. (1993) yielded the designation of radio sources labelled $\mathrm{A}$ to $\mathrm{E}$. $\mathrm{A}$ and $\mathrm{B}$ are large, extended $\left(\simeq 30^{\prime \prime}\right)$ regions of centimeter radio continuum emission, and $\mathrm{D}$ is a bright, compact radio continuum source just to the east of B. D is the southernmost component to a string of radio continuum sources that run to the northwest, ending with source $\mathrm{C}$, approximately $20^{\prime \prime}$ from D. The HMC, which lies in this string and is nearest to D, was first observed in thermal ammonia line emission by Cesaroni et al. (1994) and has been given the designation F. Masers of several species $\left(\mathrm{H}_{2} \mathrm{O}, \mathrm{OH}, \mathrm{CH}_{3} \mathrm{OH}\right.$, and $\left.\mathrm{NH}_{3}\right)$ lie along this string of radio sources, and the HMC is coincident with several water masers.

This region was previously observed by De Buizer, Piña, \& Telesco (2000) in the mid-infrared and a bright, compact source was found at both 10.5 and 18.1 $\mu \mathrm{m}$, as well as extended emission from His region B. The bright mid-infrared source was given the designation G9.619+0.193:DPT00 1 (Figure 1). Lacking accurate astrometry and given the close proximity of G9.619+0.193:DPT00 1 with the extended emission to the east, De Buizer, Piña, \& Telesco (2000) assumed that G9.619+0.193:DPT00 1 was mid-infrared emission from radio source D. Recently, Persi et al. (2003) claim that the astrometry of their mid-infrared images shows G9.619+0.193:DPT00 1 to be coincident with the location of the HMC. They established their astrometry by measuring the offset from the centroid of the mid-infrared emission from source $B$ to the location of G9.619+0.193:DPT00 1. They then found the absolute position of G9.619+0.193:DPT00 1 by applying this measured offset to the published centroid of B as given in the MSX satellite catalog. They quote an optimistic uncertainty of $1-2^{\prime \prime}$ using this method, and they state that the near-infrared emission they have observed is also coming from the HMC. A similar claim of near-infrared emission from the HMC was made previously by Testi et al. (1998).

The MIRLIN observation of this region presented in Figures 1,2 , and 3 show something different than the previous mid-infrared observations of De Buizer, Piña, \& Telesco (2000) or Persi et al. (2003). As can be seen in Figures 1 and 2 , the MIRLIN observations at $11.7 \mu \mathrm{m}$ revealed not only sources G9.619+0.193:DPT00 1 and B, but C and E as well. Figure 2 shows an overlay with the $3.5 \mathrm{~cm}$ radio continuum image of Phillips et al. (1998), which has a comparable field of view. Given the comparable resolutions and fields of view, as well as the fact that there are multiple sources in the field, positive identification can be made for all mid-infrared sources in the field. Figure 2 shows that the radio continuum and mid-infrared emission from the extended source B have similar peaks and extent. The radio continuum and mid-infrared peaks also coincide for sources $\mathrm{C}$ and $\mathrm{E}$, as well as a source (here named G9.62+0.19:DRT03 9) which lies $\sim 8^{\prime \prime}$ southeast of radio source D. However, one can see (Figures 2 and 3) that the position of G9.619+0.193:DPT00 1, given this extremely accurate registration, does not correspond spatially to either the HMC location (F) or the peak of radio source D. Given the remarkably close coincidence of midinfrared and radio continuum emission for sources B, C, E, and G9.62+0.19:DRT03 9, we claim the relative astrometry between the two wavelengths has a realistic uncertainty of $<1.0^{\prime \prime}$.

Figure 3 compares the higher resolution radio continuum and ammonia line emission observations from Hofner et al. (1994) with our mid-infrared image. One can see clearly that the mid-infrared emission of G9.619+0.193:DPT00 1 lies in between the compact radio continuum source $\mathrm{D}$ and the extended area of thermal ammonia emission $\mathrm{F}$ thought to encompass the HMC. Figure 4 shows the Keck $18.1 \mu \mathrm{m}$ image of G9.619+0.193:DPT00 1 overlaid with the radio continuum of Testi et al. (2000) and the thermal ammonia emission of Hofner et al. (1994). The Keck image was registered with the peak location of G9.619+0.193:DPT00 1 in the IRTF image. It can be seen that there is no midinfrared emission detected at Keck at the location of the $\mathrm{HMC}$ at a $3-\sigma$ upper limit of $48 \mathrm{mJy}$ at $18.1 \mu \mathrm{m}$.

Given the source crowding in this small region, it is most likely that the near infrared emission seen by Persi et al. (2003) is coming from the same source as the mid-infrared emission rather than the HMC location.

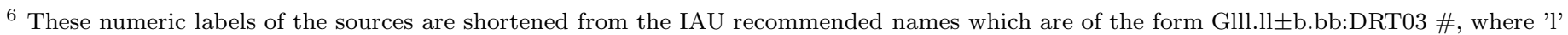

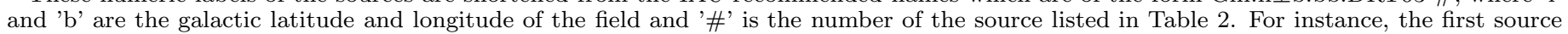
of listed in Table 2 is G9.62+0.19:DRT03 1. 
TABLE 1

HMCs AND HMC CANDidates OBSERVEd

\begin{tabular}{|c|c|c|c|c|c|c|c|c|c|}
\hline Site & $\begin{array}{l}\text { Maser } \\
\text { Group }^{\dagger}\end{array}$ & $\begin{array}{c}\mathrm{RA} \\
(\mathrm{B} 1950)\end{array}$ & $\begin{array}{c}\text { Dec } \\
(\mathrm{B} 1950)\end{array}$ & $\begin{array}{c}\mathrm{RA} \\
(\mathrm{J} 2000)\end{array}$ & $\begin{array}{c}\text { Dec } \\
(\mathrm{J} 2000)\end{array}$ & $\begin{array}{c}F_{10.5 \mu \mathrm{m}} \\
(\mathrm{mJy})\end{array}$ & $\begin{array}{c}F_{11.7 \mu \mathrm{m}} \\
(\mathrm{mJy})\end{array}$ & $\begin{array}{c}F_{18.1 \mu \mathrm{m}} \\
(\mathrm{mJy})\end{array}$ & $\begin{array}{c}F_{20.8 \mu \mathrm{m}} \\
(\mathrm{mJy})\end{array}$ \\
\hline G9.62+0.19 & 7 & 180316.08 & -203159.2 & $1806 \quad 14.82$ & -203138.4 & $<15^{\mathrm{c}}$ & $<25$ & $<48^{\mathrm{k}}$ & \\
\hline G11.94-0.62 & 3 & $\begin{array}{lll}18 & 11 & 03.70\end{array}$ & $-1854 \quad 18.4$ & $\begin{array}{lll}18 & 14 & 00.27\end{array}$ & -185323.6 & $\ldots$ & 180 & $\ldots$ & 829 \\
\hline G12.21-0.10 & 2 & $\begin{array}{lll}18 & 09 & 43.76\end{array}$ & -182506.7 & $\begin{array}{lll}18 & 12 & 39.70\end{array}$ & $\begin{array}{llll}-18 & 24 & 17.8\end{array}$ & $\ldots$ & $<28$ & $\ldots$ & $<355$ \\
\hline G19.61-0.23 & 6 & 182450.05 & -115831.6 & $\begin{array}{lll}18 & 27 & 37.84\end{array}$ & -115637.1 & $<10$ & $<31$ & $<17^{\mathrm{g}}$ & $<308$ \\
\hline $\mathrm{G} 34.26+0.15$ & 2 & $1850 \quad 46.28$ & +011112.5 & 185318.62 & +011458.1 & $<4^{\mathrm{g}}$ & $<$ ? & $<21^{\mathrm{g}}$ & $<$ ? \\
\hline G45.07-0.13 & 2 & $\begin{array}{lll}19 & 11 & 00.41\end{array}$ & +104543.3 & 191322.01 & +105053.8 & $4260^{\ddagger}$ & $5500^{\ddagger}$ & $\ldots$ & $19700^{\ddagger}$ \\
\hline G75.78+0.34 & 1 & 201951.88 & +371700.3 & $\begin{array}{lll}20 & 21 & 43.92\end{array}$ & +372638.0 & $\cdots$ & $<26$ & $\cdots$ & $<286$ \\
\hline
\end{tabular}

Note. - All 10.5 and $18.1 \mu \mathrm{m}$ data were taken with OSCIR, and all 11.7 and $20.8 \mu \mathrm{m}$ data were taken with MIRLIN. All data are from the IRTF unless otherwise noted. All values quoted with a " $<$ " are upper limit flux densities at a $95 \%$ confidence level. Flux density errors are taken to be $10 \%$ at 10.5 and $11.7 \mu \mathrm{m}$ and $15 \%$ at 18.1 and $20.8 \mu \mathrm{m}$ unless otherwise noted.

${ }^{\dagger}$ Maser group designation from Hofner \& Churchwell (1996) that is at the HMC location, or the assumed maser location of the HMC candidate. ${ }^{c}$ CTIO data from De Buizer, Piña, \& Telesco (2000).

${ }^{g}$ Gemini data.

${ }^{k}$ Keck II data.

?Even if the HMC was detected in the higher resolution Gemini images, the IRTF/MIRLIN images of this region would not be of sufficient resolution to resolve the HMC from source C.

¥There is some overlap of the extended emission from a nearby source with the HMC emission. The sources are resolved enough that a value can be estimated, however we estimate the error in the flux densities to be $5 \%$ higher in each filter.

\section{2. $\quad$ G11.94-0.62}

This site is an analog to G29.96-0.02 in that it has a cometary shaped UC His region present, with isolated water masers located out in front of the cometary arc (Hofner \& Churchwell 1996). There are two clusters of water masers near the UC HiI region, and both are offset to the west. Maser group 2 lies $\sim 2^{\prime \prime}$ from the radio continuum peak of the UC HII region, and therefore it was expected that it would be difficult to image emission of a hot molecular core separate from the emission of the UC HII in the mid-infrared, if one existed there. Water maser group 3 , however, is located $\sim 10^{\prime \prime}$ from the UC His region peak, and therefore represents a good hot molecular core candidate that can be searched for in the mid-infrared.

Figures 5 and 6 show the MIRLIN observations of this site at 11.7 and $20.8 \mu \mathrm{m}$, respectively. Interestingly, the cometary shape seen in the $\mathrm{cm}$ radio continuum images of Hofner \& Churchwell (1996) is not evident in the midinfrared (Figure 7). This is also the case for the $2.7 \mathrm{~mm}$ images of Watt \& Mundy (1999). The astrometry for this particular image is fairly well determined, since pointing of the telescope was checked immediately before and after the observations and was found to be reproducible to better than $1^{\prime \prime}$. Therefore, the overlay of the image by Hofner \& Churchwell (1996) in Figure 7 is considered to be at least that accurate. The goodness of the astrometry is further indicated by several matching structures in both the mid-infrared and radio continuum, however the radio peak is surprisingly not present in the mid-infrared.

As can be seen in Figure 7, we detected a mid-infrared source at the location of water maser group 3. Flux densities for this source at 11.7 and $20.8 \mu \mathrm{m}$ are given in Table 1. The mid-infrared emission from this candidate HMC is compact and unresolved, and exactly coincident with the water masers.

G11.94-0.62 has not yet been imaged in molecular line emission (e.g. ammonia) with sub-arcsecond resolution. The $\sim 3^{\prime \prime}$-resolution $2.7 \mathrm{~mm}$ images by Watt \& Mundy (1999) of this site do not show any dust continuum emission towards the location of maser group 3. They also failed to detect molecular $\mathrm{CH}_{3} \mathrm{CN}$ or ${ }^{13} \mathrm{CO}$ emission in their imaging of this site, even though both were detected spectroscopically by Churchwell, Walmsley, \& Wood (1992) along with CS emission. Watt \& Mundy (1999) speculate that, because of the array configuration of their interferometric observations, they may not have detected the molecular emission found by Churchwell, Walmsley, \& Wood (1992) if it is extended over a large emitting region. Cesaroni, Walmsley, \& Churchwell (1992) also detected $\mathrm{NH}_{3}$ line emission towards G11.94-0.62 spectroscopically, but it cannot be ascertained whether the $\mathrm{NH}_{3}$ is coming from an HMC at the location of water maser group 3, or the UC HII region, or both. It is encouraging that the molecular tracers, especially those from hot and dense gas (e.g. high-excitation $\mathrm{NH}_{3}$ ) are present in the region, implying the presence of an HMC on the field. However, it is disconcerting that Watt \& Mundy (1999) do not see the HMC candidate in their molecular line imaging of $\mathrm{CH}_{3} \mathrm{CN}$, which is thought to be an excellent tracer of HMCs.

\subsection{G12.21-0.10}

This is the site of another cometary UC HiI region with several water maser groups out in front of the cometary arc. This region has been observed to contain several tracers of hot and dense chemically enriched gas, such as highexcitation $\mathrm{NH}_{3}$ (Cesaroni, Walmsley, \& Churchwell 1992; Anglada et al. 1996), $\mathrm{CH}_{3} \mathrm{CN}$ (Millar \& Hatchell 1997), and CS (Plume, Jaffe, \& Evans 1992), thus making it a prime HMC candidate. However, there are not yet any direct molecular line images of this site.

Observations of G12.21-0.10 using MIRLIN did not reveal the presence of mid-infrared emission from an HMC at the location of the water masers at either 11.7 or $20.8 \mu \mathrm{m}$. Even more surprising is that the UC HII region, detected at $\mathrm{cm}$ radio wavelengths (Hofner \& Churchwell 1996) and in the submillimeter (Hatchell et al. 2000), was not detected at either mid-infrared wavelength using MIRLIN. 
The molecular and ionized environment of G19.61-0.23 was extensively studied by Garay et al. (1998). This site was found to contain five distinct Hit regions seen in radio continuum emission (labelled A, B, CD, E and F). Additionally, Garay et al. (1998) found three ammonia clumps in the region. Water and $\mathrm{OH}$ masers were discovered to be associated with the middle and most prominent of the three ammonia clumps.

In the work by De Buizer (2000, 2003), OSCIR/IRTF observations revealed the presence of mid-infrared sources coincident with all of the Hir regions of Garay et al. (1998) except source F. Figure 8 shows the $18.1 \mu \mathrm{m}$ observations of De Buizer (2000) overlaid with the radio continuum of Hofner \& Churchwell (1996). The registration between these overlaid images was performed by matching the inner radio continuum contours of Garay et al. (1998) with those of Hofner \& Churchwell (1996), which match well. Then the outer radio continuum contours of Garay et al. (1998), which are well matched with the IRTF mid-infrared contours, were used to register the mid-infrared image to the Hofner \& Churchwell (1996) image. The maser positions are known to be accurate to 0 ". 1 with respect to the radio continuum. The fact that there are four mid-infrared sources in common with the radio continuum sources of Garay et al. (1998) suggests that the astrometry is indeed accurate. Therefore the relative astrometric accuracy between the mid-infrared and the radio continuum images is believed to be $\lesssim 1$ 1. 5 .

In Figure 8 one can see that there is a weak, mid-infrared source coincident with the location of water maser group 6 from Hofner \& Churchwell (1996). This source was not detected at $10.5 \mu \mathrm{m}$, and was a $3-\sigma$ detection with a quoted flux density of $230 \pm 30 \mathrm{mJy}$ at $18.1 \mu \mathrm{m}$ (De Buizer 2000). However, this mid-infrared emission was considered to be real given the coincidence of the source with the water masers and the fact that this group of water masers exists within the confines of the middle ammonia clump of Garay et al. (1998) (Figure 9). Based on this coincidence of ammonia and water masers, De Buizer (2000) argued that this source is indeed an HMC.

Follow-up observations were performed at Gemini North using OSCIR and did not confirm the presence of a source at the location of water maser group 6 (Figure 9). A 3- $\sigma$ upper limit on the $18.1 \mu \mathrm{m}$ flux density of a source in this area from these new observations is 11 times less than the quoted flux density for this source from De Buizer (2000).

\section{5. $\quad$ G34.26+0.15}

G34.26+0.15 is a star forming complex that has been the subject of a wealth of observations at several wavelengths. It is outside the scope of this paper to discuss all of the aspects of how the new mid-infrared observations presented here complement the previous observations for all sources in this region. Such discussion will be saved for a future paper. We will, however, discuss here observations that pertain to the HMC, and we will briefly touch on certain issues regarding the other sources in the field.

Radio continuum observations by Hofner \& Churchwell (1996) show this to be a site of another cometaryshaped UC His region with water masers out in front of the cometary arc. These observations show the same radio continuum sources found by Gaume, Fey, \& Claussen
(1994), but with less extended emission than they see. Gaume, Fey, \& Claussen (1994) named the cometary UC Hir region source $\mathrm{C}$, and the two unresolved bright UC HiI regions located east and in front of the cometary arc as $\mathrm{A}$ and B (southern and northern sources, respectively; see Figure 10). In front of the cometary arc of $\mathrm{C}$, there is a confirmed HMC that is seen in several molecular species (e.g. Keto et al. 1992; Churchwell, Walmsley, \& Wood 1992).

The most complete mid-infrared observations of this region were performed by Campbell et al. (2000) at the IRTF. They observed the region through six mid-infrared filters from 8.0 to $20.6 \mu \mathrm{m}$ with $1^{\prime \prime} .2-1^{\prime \prime} .7$ resolution. Like the earlier mid-infrared observations of Keto et al. (1992) of this site, Campbell et al. (2000) found the mid-infrared emission of radio continuum source $\mathrm{C}$ not to be cometary in the mid-infrared, and failed to detect emission from radio continuum source B. However, Campbell et al. (2000) did discover two new sources seen only at $20.6 \mu \mathrm{m}$, one of which, designated E, lies just south of source C. Keto et al. (1992) also claimed to have discovered an additional midinfrared source at $12.5 \mu \mathrm{m}$ at the location of the HMC with a flux density of $70 \mathrm{mJy}$. Campbell et al. (2000) did not detect this source, but they could not discount its existence given the noise level of their images.

One problem with comparing the radio continuum images of $\mathrm{G} 34.26+0.15$ with our images is that the separation between sources $\mathrm{A}$ and $\mathrm{C}$ is $0 . .5$ larger in the radio than in our mid-infrared images. Campbell et al. (2000) notes this as well, stating that registering their mid-infrared images on radio source A resulted in an offset of 0.5 in the peak locations of $\mathrm{C}$ that did not appear to be real. They concluded that the lower signal-to-noise ratio of $\mathrm{A}$ was likely to be the cause of the scatter, and they therefore registered all of their mid-infrared images using the radio continuum peak of $\mathrm{C}$. In our mid-infrared images source A has good signal-to-noise, yet we have drawn the same conclusion that the mid-infrared morphology of source $\mathrm{C}$ is too similar to the radio morphology for the offset to be real. Therefore, like Campbell et al. (2000), we registered our mid-infrared images using source C. Surprisingly, the $12.5 \mu \mathrm{m}$ image of Keto et al. (1992) shows sources A and $\mathrm{C}$ to have the same separation as the radio continuum sources, inconsistent with the conclusion by Campbell et al. (2000) and our results here. This uncertainty in registration, however, does not affect our following interpretation of the region.

Figures 11 and 12 show the 10.5 and $18.1 \mu \mathrm{m}$ OSCIR images from Gemini North. Two things are immediately obvious from these figures. First, radio continuum source $\mathrm{C}$ does not look like a single stellar source with a cometary shaped UC Hı region in the mid-infrared. Instead it consists of perhaps four individual components (not two, C and E, as suggested by Campbell et al. 2000; see Table 2). Second, the mid-infrared emission from the HMC is absent in both images. This can best be seen in Figure 13 which shows the Gemini $10.5 \mu \mathrm{m}$ image overlaid with the 12.5 $\mu \mathrm{m}$ image of Keto et al. (1992). Figure 14 shows the $\mathrm{NH}_{3}$ image of Keto et al. (1992) overlaid on the $18.1 \mu \mathrm{m}$ image from Gemini for comparison. The $\mathrm{NH}_{3}$ peak is actually a little south of the $12.5 \mu \mathrm{m}$ peak, however the Gemini observations show that there are no signs of mid-infrared 
emission from either location or from the location of any of the other isolated water maser groups in the region. These Gemini observations cast doubt on the existence of the mid-infrared source seen by Keto et al. (1992), since the 3- $\sigma$ upper limit on the presence of such a source is 4 $\mathrm{mJy}$ at $10.5 \mu \mathrm{m}$ and $21 \mathrm{mJy}$ at $18.1 \mu \mathrm{m}$.

Keto et al. (1992) also presented one other mid-infrared detection of an HMC at the location of W3 $\left(\mathrm{H}_{2} \mathrm{O}\right)$. Similar to the result presented here, this other HMC was not detected in the recent observations by Stecklum et al. (2002), who give an upper limit far below the flux density quoted by Keto et al. (1992).

\section{6. $\quad$ G45.0\%-0.13}

The observations of Hofner \& Churchwell (1996) show this site to contain an unresolved, bright UC HII region coincident with several water masers. There is, however, one tight group of water masers offset $\sim 2^{\prime \prime}$ north of the UC HII peak. Given the compact nature of the UC HII region in the radio, the northern group of water masers was believed to be far enough away to distinguish mid-infrared emission from an HMC, if one exists there.

There exist no high-spatial resolution molecular line images of this site. However, like G11.94-0.62, this region has been observed to contain tracers of hot and dense chemically enriched gas, such as $\mathrm{CH}_{3} \mathrm{CN}$ (Pankonin et al. 2001). The mid-infrared images presented in Figures 15 and 16 are 11.7 and $20.8 \mu \mathrm{m}$ images, respectively, from MIRLIN at then IRTF. These images show three mid-infrared sources in the area. The middle and brightest of these mid-infrared sources is closest to the location of the UC HII region and assumed to be coincident. Figure 17 shows the $11.7 \mu \mathrm{m}$ image overlaid with the radio continuum contours and masers from Hofner \& Chuchwell (1996). In this figure one can see that the location of the isolated northern water maser group is exactly coincident with the northern mid-infrared source.

G45.07-0.13 has also been imaged at $3 \mathrm{~mm}$ by Hunter, Phillips, \& Menten (1997). They see emission from the UC HII region as well as the southern mid-infrared source. Given the resolution of the $3 \mathrm{~mm}$ images, it is difficult to say whether or not they should be able to resolve the emission from the HMC candidate at the northern water maser location. The HMC candidate may be faint at 3 $\mathrm{mm}$, or unresolved from the UC Hit region, or both.

The water masers associated with the UC HiI region, as discussed in Hofner \& Churchwell (1996), lie in a linear distribution at position angle of $-45^{\circ}$. This is a similar position angle to the axis of an outflow seen at this location by Hunter, Phillips, \& Menten (1997) observed in CO and CS. Hofner and Churchwell (1996) suggest that the water masers and outflow are related, thus implying that the outflows here emanate from the UC HiI region. Hunter, Phillips, \& Menten (1997) also claim that the outflow is driven by a single isolated stellar source at the center of the UC Hir region. However, given the resolution of the $\mathrm{CO}$ and CS images, any of the three sources,including the HMC candidate, seen in the mid-infrared are candidates for the outflow source.

3.7. $G 75.78+0.34$
The UC HiI region present here is coincident with the $\mathrm{OH}$ maser ON2-N, and therefore this site is also known by that name. Furthermore, this site is also known as G75.780.34 , as a result of a misprint in the paper by Hofner \& Churchwell (1996). At radio continuum wavelengths, Hofner \& Churchwell (1996) find the UC His emission to be cometary shaped, again with water masers offset and located in front of the cometary arc by $\sim 2^{\prime \prime}$.

G75.78+0.34 has never been imaged in molecular line emission, however it is the site of a variety of molecules known to be tracers of high density material associated with HMCs. Tracers like $\mathrm{CH}_{3} \mathrm{CN}$ (Hatchell et al. 1998) and CS (Olmi \& Cesaroni 1999) have been detected here, as well as high excitation lines of $\mathrm{NH}_{3}$ (Olmi, Cesaroni, \& Walmsley 1993). However, the best evidence for the presence of a compact object at the location of the water masers is the detection of a $7 \mathrm{~mm}$ source at the maser location by Carral et al. (1997). Emission was also detected by Carral et al. (1997) at wavelengths out to $2 \mathrm{~cm}$. Given the extremely high emission measure of this object, it has been deemed a "hypercompact HiI region" (Kurtz \& Franco 2002), and as such is thought to represent a stellar phase much younger than the UC Hir phase.

Despite the fact that this appears to be an excellent candidate for mid-infrared study, we failed to detect any mid-infrared emission at either 11.7 or $20.8 \mu \mathrm{m}$ with MIRLIN at IRTF from the location of the water masers. Even more surprisingly, like G12.21-0.10, the UC HII region was not detected either. Upper limits for flux densities for these objects are given in Table 1.

\section{PHYSICAL PROPERTIES OF THE HMC CANDIDATES}

We have derived estimates of several physical parameters for the newly detected HMC candidates seen in G11.94-0.62 and G45.07-0.13. A detailed discussion of how the color temperature, mid-infrared luminosity, and spectral type were derived are discussed in De Buizer, Piña, \& Telesco (2000), and the discussion of the limitations of this method are discussed in more detail in De Buizer et al. (2002b). Basically, for each source a blackbody function was fit to the 11.7 and $20.8 \mu \mathrm{m}$ flux densities. From this Planck-function fit a dust color temperature was found. Integrating this Planck function from 1 to $600 \mu \mathrm{m}$ and using this distance to the source we derived a mid-infrared luminosity. The distances to the sources were taken to be $4.2 \mathrm{kpc}$ and $6.0 \mathrm{kpc}$ for G11.94-0.62 and G45.07-0.13, respectively (Hofner \& Churchwell 1996).

These calculations yield a blackbody dust color temperature of $167 \mathrm{~K}$ for G11.94-0.62 and $181 \mathrm{~K}$ for G45.07-0.13. Because these are blackbody dust color temperature values, they can be considered upper limits on the actual mid-infrared dust color temperature. The blackbody midinfrared luminosities derived for the sources are 90 and $4360 \mathrm{~L}_{\text {sun }}$ for G11.94-0.62 and G45.07-0.13, respectively. Again, because these are blackbody mid-infrared luminosities, they are a reflection of only a part of the bolometric luminosity and therefore can be considered to be lower limits (possibly extreme lower limits) to the actual bolometric luminosity of the central heating sources. Making the simple assumption that the mid-infrared luminosities are equal to the bolometric luminosities of the sources, we can derive ZAMS spectral type limits which would be 
an estimate of the latest spectral type the central heating source could possibly be. These spectral type limits were found to be B9 and B0 for G11.94-0.62 and G45.07-0.13, respectively.

We can also derive limits to the physical size of the HMC candidates in the mid-infrared. The blackbody lower limit size calculated for G11.94-0.62 is $105 \mathrm{AU}$ and $624 \mathrm{AU}$ for G45.07-0.13. Following the procedure outlined in Soifer et al. (2000), we used seven IRTF/MIRLIN PSF observations to derive an upper limit size for the HMC candidates. The rms scatter of the PSF FWHMs was found to be 0.1 at $11.7 \mu \mathrm{m}$. Since the HMCs, like the PSF stars, are unresolved at this wavelength, we can take the value of $5-\sigma$ $(0 \prime .5)$ as the upper limit of the size of both HMC candidates under the assumption that a difference of 5 times the standard deviation of the PSF FWHM would have been easily discernable. This yields a upper limit physical size for the HMC candidates of $2100 \mathrm{AU}$ for G11.94-0.62 and 3000 AU for G45.07-0.13.

We can compare these to the only other mid-infrared bright HMC presently, G29.96-0.02. This source, however, is resolved with a PSF-quadrature-subtracted physical size of $6720 \mathrm{AU}$. This is a few times larger than the upper limits we have set on G11.94-0.62 and G45.07-0.13. Since the HMC in G29.96-0.02 is resolved, the dust color temperature will be closer to the quoted optically thin dust color temperature value of $105 \mathrm{~K}$ (De Buizer et al. 2002a) which yields a mid-infrared luminosity of $490 \mathrm{~L}_{\odot}$. This estimates the spectral type at $>$ B6, derived from the mid-infrared luminosity alone. This shows that all three HMCs are at least luminous enough to contain B-type stars, and very possibly O-type stars at there centers. This is consistent with the hypothesis that the central stars heating these mid-infrared bright HMC candidates are indeed massive, assuming that they are heated by a single source.

\section{DISCUSSION}

The main result of these observations is the detection of two new HMC candidates, which when combined with the mid-infrared detection of G29.96-0.02 (De Buizer et al. 2002a), represent three HMC candidates to be directly imaged recently in the mid-infrared from the survey of Hofner \& Churchwell (1996). Unlike G29.96-0.02, however, the HMC candidates of G11.94-0.62 and G45.07-0.13 have not been directly imaged in molecular line emission. Therefore can we be sure that these are, in fact, HMCs? There are several reasons why it is believed that these two mid-infrared sources are HMCs. First, all of the midinfrared sources have no radio continuum emission, implying that they are indeed young and embedded. Second, this survey has relatively good astrometry and both of these HMC candidates are directly coincident with clusters of water masers, which are generally believed to trace young massive stars. Third, there are spectroscopic observations providing evidence of high-excitation molecular emission line coming from each of these regions, a necessary (but not sufficient) condition if these are HMCs. Finally, we derive estimates of mid-infrared luminosities for these HMCs that are consistent with their being heated by massive central stars. Taken together, these four pieces of evidence combined lead to the belief that these are indeed HMCs. However, confirmation of the hot molecular nature of these sources will have to come from high resolution molecular line imaging (e.g. $\mathrm{NH}_{3}$ or $\mathrm{CH}_{3} \mathrm{CN}$ ).

While the mid-infrared detections of G11.94-0.62, G29.96-0.02, and G45.07-0.13 demonstrate that HMCs can indeed be imaged at mid-infrared wavelengths, our lack of confirmation of the previously reported detections of HMCs in the mid-infrared in the fields of G9.62+0.19, G19.61-0.23, and G34.26+0.15 show that one must be extremely careful in their astrometry and that one must image these sources deeply in order to confirm their existence as HMCs. Given the gregarious nature of young massive stars, the fields containing HMCs are crowded with sources at many different stages of formation. We must be careful that we are referring to the same source when comparing fields at different wavelengths, since these different wavelengths are sensitive to different stellar phases.

Of the 21 sites in the survey of Hofner \& Churchwell (1996), nine stand out as good candidates for HMCs, given the fact that they contain water masers isolated and offset from nearby UC HII regions and display evidence for molecular line emission. G43.89-0.38 was the only one of these nine sources not observed in either this work or the G29.96-0.02 article by De Buizer et al. (2002a). Of the eight sources observed from Hofner \& Churchwell (1996), we have detected mid-infrared emission from the HMCs of three sources in total. Though it is certainly small number statistics, this suggests a mid-infrared detection success rate towards isolated water masers in regions of massive star formation of $38 \%$. Why is there such a low mid-infrared detection rate for HMCs? The most obvious reason is that if the HMCs are too cold and embedded, very little mid-infrared emission would be expected. This would be the case if the HMCs are at an extremely early stage of development. This implies that HMCs that display mid-infrared emission are at a more evolved stage of formation than HMCs lacking mid-infrared emission.

Since we need to confirm the validity of the HMC through molecular line imaging anyway, one might ask why we should even image the HMCs in the mid-infrared at all. In addition to the reason just mentioned, i.e. that it gives us an idea of the relative age of the HMC, one may be able to use mid-infrared observations at more wavelengths to more accurately describe the physical characteristics of massive star formation. Models of the earliest stages of massive star formation are being produced which calculate emergent thermal dust spectral energy distributions (e.g. Osorio, Lizano, \& D'Alessio 1999). These SEDs model an $\mathrm{HMC}$ as an envelope of gas and dust that is infalling and accreting onto a massive central protostar. The models take into account the effects of mass accretion rate and the luminosity of the central proto-stellar object on the radiative transfer through the envelope as a function of the envelope size.

The simple models of Osorio, Lizano, \& D'Alessio (1999) showed a great dependence of the emergent SED at midinfrared wavelengths on the physical characteristics of the HMC. On the steep Wien side of the SED, the depth and shape of the $10 \mu \mathrm{m}$ silicate feature and the lower wavelength fall-off of emission are heavily dependent on accretion rate and central proto-stellar luminosity. In the Rayleigh-Jeans portion of the SED (i.e. at far-infrared and longer wavelengths), the shape of the SED is simply 
a constant slope that appears to be predominantly determined by the dust opacity at these wavelengths. Most models adopt a power law for the dust opacity of the form, $\kappa_{\lambda} \propto \lambda^{-\beta}$, where $1 \leq \beta \leq 2$ for $\lambda \geq 200 \mu \mathrm{m}$. Consequently, data at wavelengths longer than $\sim 100 \mu \mathrm{m}$ do not drastically effect the SED and hence do not reveal much about the physical processes, such as accretion rate. However, the molecular lines in the sub-millimeter and millimeter are still helpful for deriving the HMC temperatures (which tells one where the SED peaks) and density values needed for accurate modelling of the radiative transfer. Though such models are still in their infancy, a more complete sampling of the SED at a variety of mid-infrared wavelengths (i.e. more than the one or two wavelengths sampled here) will yield more accurate information of the physical characteristics of HMCs as the models become more refined.

\section{CONCLUSIONS}

In this paper we presented the detection of mid-infrared emission from the locations of two HMC candidates: G11.94-0.62 and G45.07-0.13. Both of these sources are believed to be genuine HMCs because they display no radio continuum emission, are directly coincident with clusters of water masers, reside in regions of high-excitation molecular emission line emission observed spectroscopically, and have derived mid-infrared luminosities that imply they are being heated internally by massive $\mathrm{B}$ or $\mathrm{O}$ type stars.

We also presented deep mid-infrared images of two HMCs previously detected in the mid-infrared: G19.610.23 and G34.26+0.15. For both of these sources we failed to detect mid-infrared emission from the HMC location at any wavelength. For G9.62+0.19, we were able to determine that the mid-infrared emission thought to be associated with the HMC is in fact offset $\sim 2^{\prime \prime}$ from the HMC location. Given the density of sources on the field, the emission seen in molecular line images and the mid-infrared emission are most likely coming from different sources altogether.

The two HMCs newly detected in the mid-infrared, along with the HMC in G29.96-0.02 (De Buizer et al. 2002a), represent a small, but growing group of sources that can be modelled to learn about the physical characteristics of massive star formation. Further observations and mid-infrared spectra of these sources will provide extremely useful information for the modelling and characterization of these youngest stages of massive stellar birth.

Data presented in this article were in part obtained at the Infrared Telescope Facility, which is operated by the University of Hawaii under a cooperative agreement with the National Aeronautics and Space Administration. This material is based upon work supported by the National Aeronautics and Space Administration under Cooperative Agreement no. NCC 5-538 issued through the Office of Space Science, Planetary Astronomy Program.

Some data were also obtained at the Gemini Observatory, which is operated by the Association of Universities for Research in Astronomy, Inc., under a cooperative agreement with the NSF on behalf of the Gemini partnership: the National Science Foundation (United States), the Particle Physics and Astronomy Research Council (United Kingdom), the National Research Council (Canada), CONICYT (Chile), the Australian Research Council (Australia), CNPq (Brazil) and CONICET (Argentina).

Further data presented herein were obtained at the W.M. Keck Observatory, which is operated as a scientific partnership among the California Institute of Technology, the University of California, and the National Aeronautics and Space Administration. The Observatory was made possible by the generous support of the W.M. Keck Foundation.

\section{REFERENCES}

Anglada, G., Estalella, R., Pastor, J., Rodriguez, L. F., \& Haschick, A. D. 1996, ApJ, 463, 205

Campbell, M. F., Garland, C. A., Deutsch, L. K., Hora, J. L., Fazio, G. G., Dayal, A., \& Hoffmann, W. F. 2000, ApJ, 536, 816

Carral, P., Kurtz, S. E., Rodriguez, L. F., de Pree, C., \& Hofner, P. 1997, ApJ, 486, L103

Cesaroni, R., Walmsley, C. M., \& Churchwell, E. 1992, A\&A, 256, 618

Cesaroni, R., Churchwell, E., Hofner, P., Walmsley, C. M., \& Kurtz, S. 1994, A\&A, 288, 903

Churchwell, E., Walmsley, C. M., \& Wood, D. O. S. 1992, A\&A, 253, 541

De Buizer, J. M. 2000, PhD Thesis, University of Florida

De Buizer, J. M. 2003, in ASP Conference Series, Vol. 287, Galactic Star Formation Across the Stellar Mass Spectrum, J. M. De Buizer \& N. S. van der Bliek, eds. (San Francisco: Astronomical Society of the Pacific), 230

De Buizer, J. M., Piña, R. K., \& Telesco, C. M. 2000, ApJS, 130, 437

De Buizer, J. M., Watson, A. M., Radomski, J. T., Piña, R. K., \& Telesco, C. M. 2002, ApJ, 564, L101

De Buizer, J. M., Radomski, J. T., Piña, R. K., \& Telesco, C. M. 2002, ApJ, 580, 305

Garay, G., Rodriguez, L. F., Moran, J. M., \& Churchwell, E. 1993, ApJ, 418, 368

Garay, G., Moran, J. M., Rodriguez, L. F., \& Reid, M. J. 1998, ApJ, 492,635

Gaume, R. A., Fey, A. L., \& Claussen, M. J. 1994, ApJ, 432, 648

Hatchell, J., Thompson, M. A., Millar, T. J., \& MacDonald, G. H. 1998, A\&AS, 133, 29
Hatchell, J., Fuller, G. A., Millar, T. J., Thompson, M. A., \& Macdonald, G. H. 2000, A\&A, 357, 637

Hofner, P., Kurtz, S., Churchwell, E., Walmsley, C. M., \& Cesaroni, R. 1994, ApJ, 429, L85

Hofner, P. \& Churchwell, E. 1996, A\&AS, 120, 283

Hunter, T. R., Phillips, T. G., \& Menten, K. M. 1997, ApJ, 478, 283

Keto, E., Proctor, D., Ball, R., Arens, J., \& Jernigan, G. 1992, ApJ, 401, L113

Kruegel, E. \& Walmsley, C. M. 1984, A\&A, 130, 5

Kurtz, S. \& Franco, J. 2002, in Revista Mexicana de Astronomía y Astrofísica Serie de Conferencias Vol. 12, Ionized Gaseous Nebulae, a Conference to Celebrate the 60th Birthdays of Silvia Torres-Peimbert and Manuel Peimbert, W. J. Henney, J. Franco, M. Martos, \& M. Peña), eds. (Morelia: UNAM), 16

Millar, T. J. \& Hatchell, J. 1997, Communications of the Konkoly Observatory, 100, 207

Olmi, L. \& Cesaroni, R. 1999, A\&A, 352, 266

Olmi, L., Cesaroni, R., \& Walmsley, C. M. 1993, A\&A, 276, 489

Osorio, M., Lizano, S., \& D'Alessio, P. 1999, ApJ, 525, 808

Pankonin, V., Churchwell, E., Watson, C., \& Bieging, J. H. 2001, ApJ, 558, 194

Persi, P., Tapia, M., Roth, M., Marenzi, A. R., Testi, L., \& Vanzi, L. 2003, A\&A, 397, 227

Phillips, C. J., Norris, R. P., Ellingsen, S. P., \& McCulloch, P. M. 1998, MNRAS, 300, 1131

Plume, R., Jaffe, D. T., \& Evans, N. J. 1992, ApJS, 78, 505

Soifer, B. T., Neugebauer, G., Matthews, K., Egami, E., Becklin, E. E., Weinberger, A. J., Ressler, M., Werner, M. W., Evans, A. S., Scoville, N. Z., Surace, J. A., Condon, J. J. 2000, ApJ, 119, 509 
Stecklum, B., Brandl, B., Henning, T., Pascucci, I., Hayward, T. L., \& Wilson, J. C. 2002, A\&A, 392, 1025

Testi, L., Felli, M., Persi, P., \& Roth, M. 1998, A\&A, 329, 233
Testi, L., Hofner, P., Kurtz, S., \& Rupen, M. 2000, A\&A, 359, L5

Watt, S. \& Mundy, L. G. 1999, ApJS, 125, 143 
TABLE 2

Mir Flux Densities for All Sources Observed in Target Field of View

\begin{tabular}{|c|c|c|c|c|c|c|c|}
\hline Field & Source $^{\dagger}$ & $\begin{array}{c}\Delta \mathrm{RA}^{\dagger} \\
\left(^{\prime \prime}\right)\end{array}$ & $\begin{array}{c}\Delta \operatorname{Dec}^{\dagger} \\
\left({ }^{\prime \prime}\right)\end{array}$ & $\begin{array}{c}F_{10.5 \mu \mathrm{m}} \\
(\mathrm{Jy})\end{array}$ & $\begin{array}{c}F_{11.7 \mu \mathrm{m}} \\
(\mathrm{Jy})\end{array}$ & $\begin{array}{c}F_{18.1 \mu \mathrm{m}} \\
(\mathrm{Jy})\end{array}$ & $\begin{array}{c}F_{20.8 \mu \mathrm{m}} \\
(\mathrm{Jy})\end{array}$ \\
\hline \multirow{9}{*}{ G9.62+0.19 } & 1 & -20.8 & +10.3 & $\cdots$ & 0.20 & $\cdots$ & $\cdots$ \\
\hline & 2 & -14.5 & +11.8 & $\ldots$ & 1.56 & $\ldots$ & $\ldots$ \\
\hline & 3 & -8.6 & -7.8 & $\ldots$ & 18.6 & $\ldots$ & $\ldots$ \\
\hline & 4 & -6.8 & +12.5 & $\ldots$ & 0.34 & $\ldots$ & $\ldots$ \\
\hline & 5 & -5.2 & +6.9 & $\cdots$ & 0.14 & $\ldots$ & $\cdots$ \\
\hline & 6 & -2.6 & +6.5 & ... & 0.17 & $\ldots$ & $\ldots$ \\
\hline & 7 & -1.8 & +13.2 & $\ldots$ & 0.24 & $\ldots$ & $\ldots$ \\
\hline & $8^{\ddagger}$ & +1.6 & -2.6 & $0.33^{\mathrm{c}}$ & 0.43 & $3.17^{\mathrm{c}}$ & $\ldots$ \\
\hline & 9 & +4.1 & -12.3 & $\ldots$ & 0.07 & $\ldots$ & \\
\hline \multirow{7}{*}{ G11.94-0.62 } & 1 & +0.5 & +0.5 & $\ldots$ & 0.18 & $\ldots$ & 0.83 \\
\hline & 2 & +8.6 & +0.8 & $\ldots$ & $1.11^{?}$ & $\ldots$ & $5.43^{?}$ \\
\hline & 3 & +10.8 & +5.0 & $\ldots$ & $1.28 ?$ & $\ldots$ & $7.82^{?}$ \\
\hline & 4 & +11.1 & -2.3 & . & $1.44^{?}$ & $\cdots$ & $11.3^{?}$ \\
\hline & 5 & +11.7 & +0.1 & $\ldots$ & $1.45^{?}$ & $\cdots$ & $11.1^{?}$ \\
\hline & 6 & +17.3 & -3.3 & $\ldots$ & $1.21^{?}$ & $\ldots$ & $8.70^{?}$ \\
\hline & 7 & +17.7 & -7.2 & $\ldots$ & $<0.03$ & $\ldots$ & 5.01 \\
\hline \multirow{6}{*}{ G19.61-0.23 } & 1 & -11.4 & +9.0 & $<0.01$ & 0.58 & 1.39 & 3.66 \\
\hline & 2 & -8.0 & +5.1 & 2.64 & 3.04 & 9.20 & 17.4 \\
\hline & 3 & -3.5 & -3.6 & 9.47 & 11.6 & 52.8 & 82.9 \\
\hline & 4 & +5.6 & +9.4 & 4.06 & $3.36^{?}$ & 18.3 & $33.5^{?}$ \\
\hline & 5 & +12.6 & -8.1 & 4.02 & $4.75 ?$ & 21.2 & $20.9^{?}$ \\
\hline & 6 & +16.8 & -5.6 & $<0.01$ & $<0.03$ & 1.08 & 2.70 \\
\hline \multirow[t]{5}{*}{ G34.26+0.15 } & 1 & -2.4 & -1.2 & $0.24^{? g}$ & $U$ & $0.64^{? g}$ & $U$ \\
\hline & 2 & -2.1 & -2.1 & $0.15^{? g}$ & $U$ & $1.24^{? g}$ & $U$ \\
\hline & 3 & -1.9 & -0.4 & $0.51^{? g}$ & $U$ & $0.75^{? g}$ & $U$ \\
\hline & 4 & -1.3 & +0.2 & $3.53^{? g}$ & $U$ & $3.68^{? g}$ & $U$ \\
\hline & 5 & +1.6 & -1.4 & 0.81 & 0.61 & 2.06 & 5.11 \\
\hline \multirow[t]{3}{*}{ G45.07-0.13 } & 1 & -3.9 & -7.3 & 0.89 & 1.40 & $\cdots$ & 15.1 \\
\hline & 2 & 0.0 & -2.2 & 20.4 & $21.7^{?}$ & $\ldots$ & $19.7^{?}$ \\
\hline & 3 & +0.2 & 0.0 & 4.26 & $5.50^{?}$ & $\ldots$ & $102^{?}$ \\
\hline
\end{tabular}

Note. - All 10.5 and $18.1 \mu \mathrm{m}$ data were taken with OSCIR, and all 11.7 and $20.8 \mu \mathrm{m}$ data were taken with MIRLIN. All data are from the IRTF unless otherwise noted. All values quoted with a "<" are upper limit flux densities at a $95 \%$ confidence level. Flux density errors are taken to be $10 \%$ at 10.5 and $11.7 \mu \mathrm{m}$ and $15 \%$ at 18.1 and $20.8 \mu \mathrm{m}$ unless otherwise noted.

${ }^{\dagger}$ Mid-infrared sources are numbered from west to east on each field. The positions of the sources are given as offsets in arcseconds from the maser/HMC location given in Table 1.

${ }^{\ddagger}$ G9.619+0.193:DPT00 1 from De Buizer, Piña, \& Telesco (2000).

${ }^{c}$ CTIO data from De Buizer, Piña, \& Telesco (2000).

${ }^{g}$ Gemini data.

? There is some overlap of the extended emission from a nearby source. The sources are resolved enough that a value can be estimated, however we estimate the error in the flux densities to be $5 \%$ higher in each filter.

${ }^{U}$ The source was not sufficiently resolved from a nearby source enough to estimate a flux density measurement. 


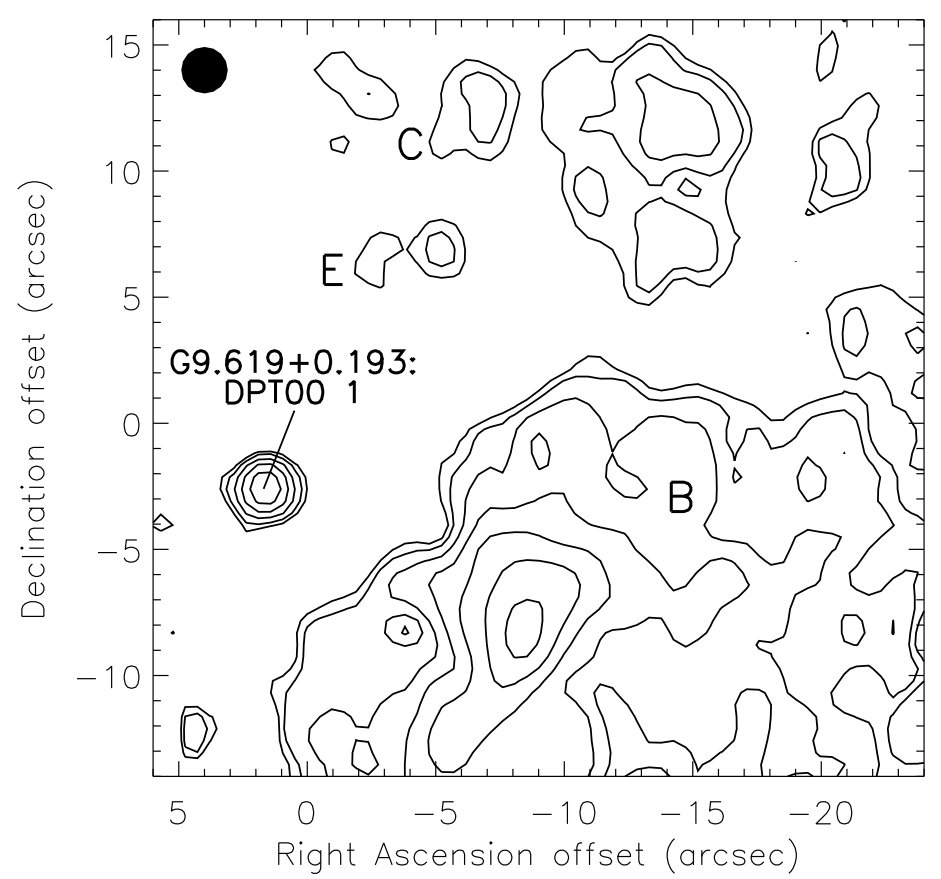

FIG. 1.- Contour plot of the MIRLIN/IRTF $11.7 \mu \mathrm{m}$ image of the G9.62+0.19 region. Labelled are the mid-infrared components radio sources as defined by Garay et al. (1993). Also labelled is the mid-infrared source G9.619+.193:DPT00 1. The filled circle in the upper left represents the FWHM resolution of the image.

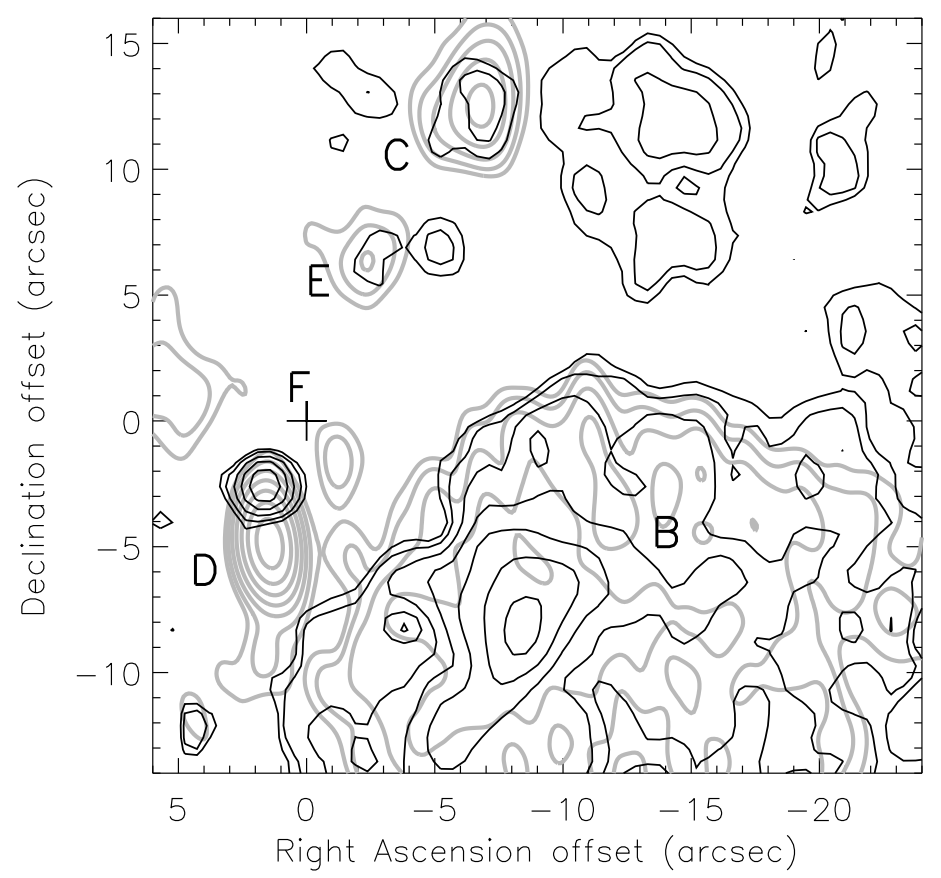

FIG. 2.- A comparison between the $3.5 \mathrm{~cm}$ radio continuum image (gray contours) of Phillips et al. (1998) with the $11.7 \mu \mathrm{m}$ IRTF image (black contours) taken with MIRLIN for the region around G9.62+0.19. Radio components as defined by Garay et al. (1993) are labelled. Sources C and E are imaged here for the first time in the mid-infrared. Along with the extended emission of source B, these sources finally and accurately anchor the astrometry between the radio continuum and mid-infrared. Radio source A is off-field and west of B. The position of the ammonia source center is shown as a cross and labelled F. 


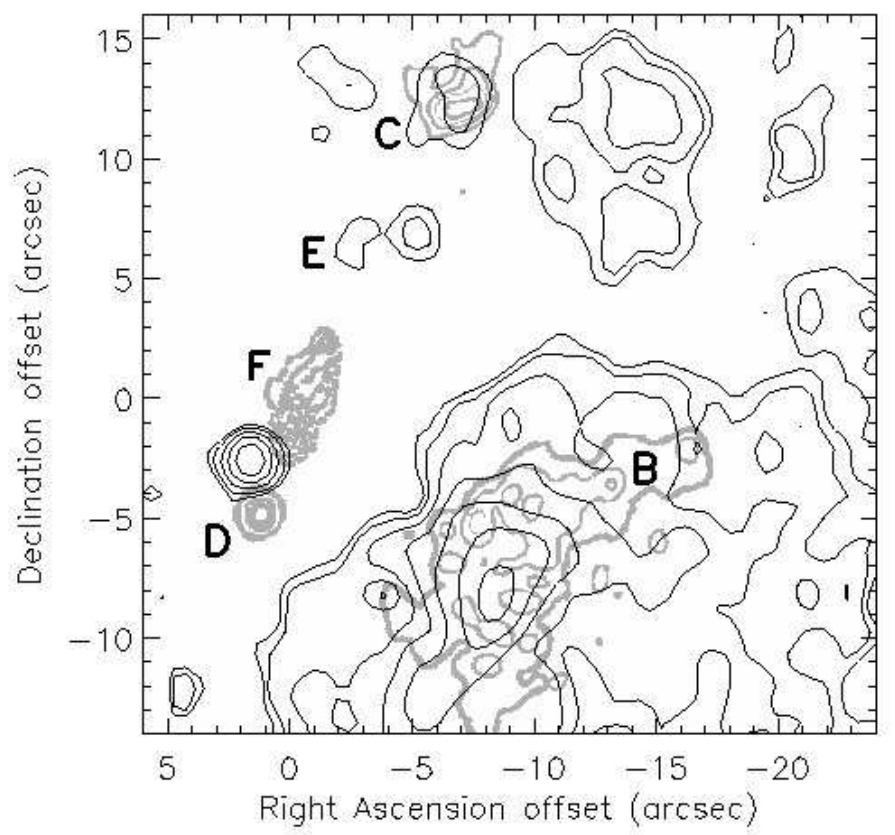

FIG. 3. - A comparison between the $11.7 \mu \mathrm{m}$ MIRLIN/IRTF image (black contours) and Figure 4 from Hofner et al. (1994) for G9.62+0.19. The gray continuous contours are high-resolution $3.6 \mathrm{~cm}$ radio continuum emission, and the gray broken contours delineate the areas of thermal $\mathrm{NH}_{3}(5,5)$ emission. In this image it can be clearly seen that the compact and bright mid-infrared source G9.619+0.193:DPT00 1 is not coincident with either the radio continuum source D nor the hot molecular core, $\mathrm{F}$, seen in thermal ammonia.

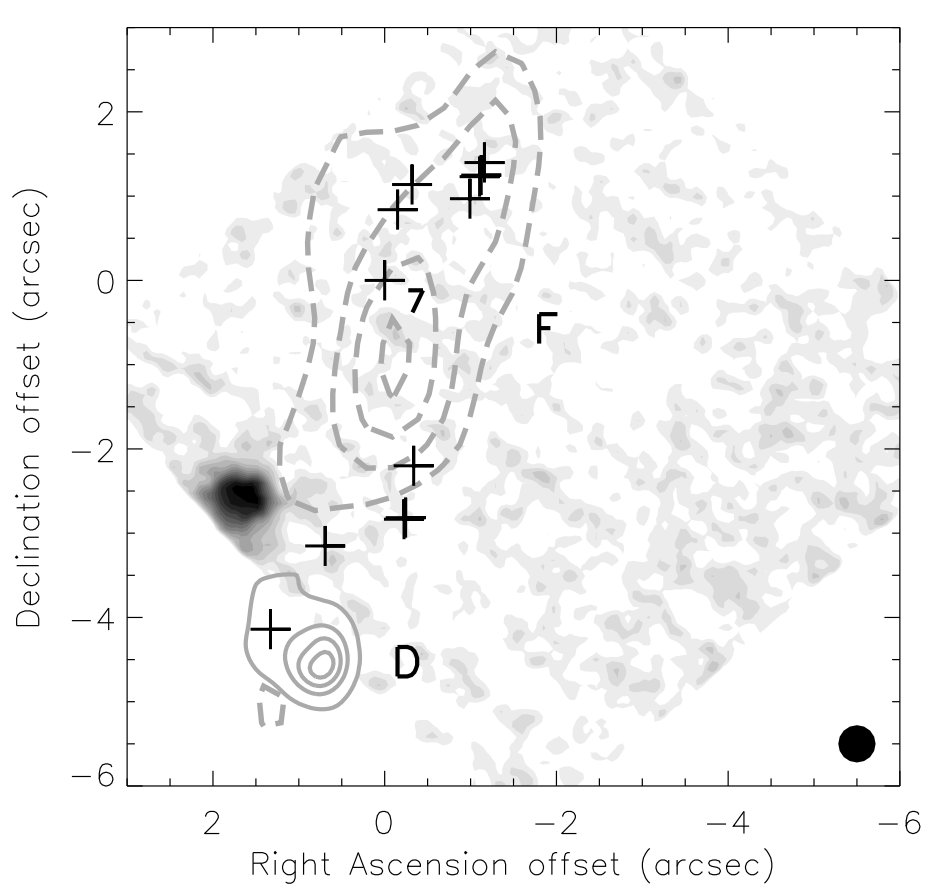

FIG. 4. - The OSCIR/Keck $18.1 \mu \mathrm{m}$ image (gray-scale) of G9.62+0.19 overlaid with the thermal ammonia emission (gray broken contours) of Hofner et al. (1994) of source F, and the $3.6 \mathrm{~cm}$ radio continuum emission (gray continuous contours) of source D from Testi et al. (2000). Also shown are the water maser group locations (crosses) from Hofner \& Churchwell (1996). Water maser group 7 is labelled and is the closest to the HMC center. No mid-infrared source was detected at the maser/HMC location with a 3- $\sigma$ upper limit value of $48 \mathrm{mJy}$ at $18.1 \mu \mathrm{m}$. The filled circle in the lower right represents the FWHM resolution of the mid-infrared Keck image. 


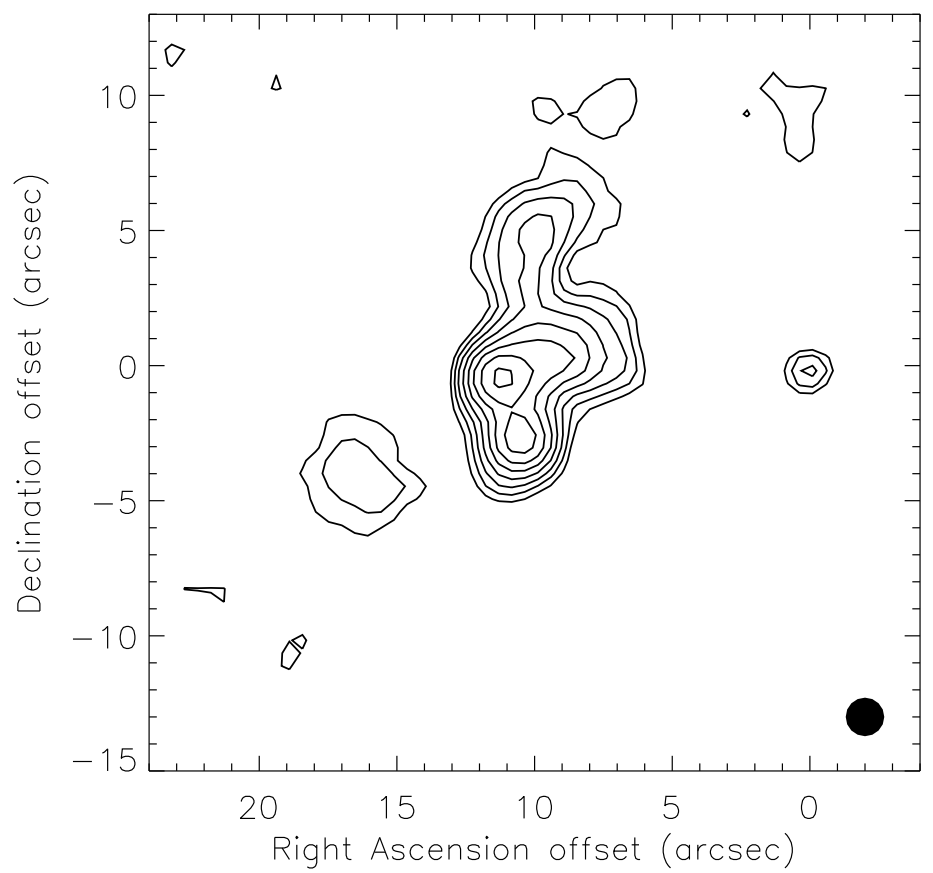

FIG. 5.- A contour plot of the MIRLIN/IRTF $11.7 \mu \mathrm{m}$ image of G11.94-0.62. The compact, unresolved source at the origin is the HMC candidate. The cometary UC HII region in this field does not appear to have a cometary shape in the mid-infrared. Instead it can be seen as the group of close and spatially unresolved sources to the east of the HMC location. The filled circle in the lower right represents the FWHM resolution of the image.

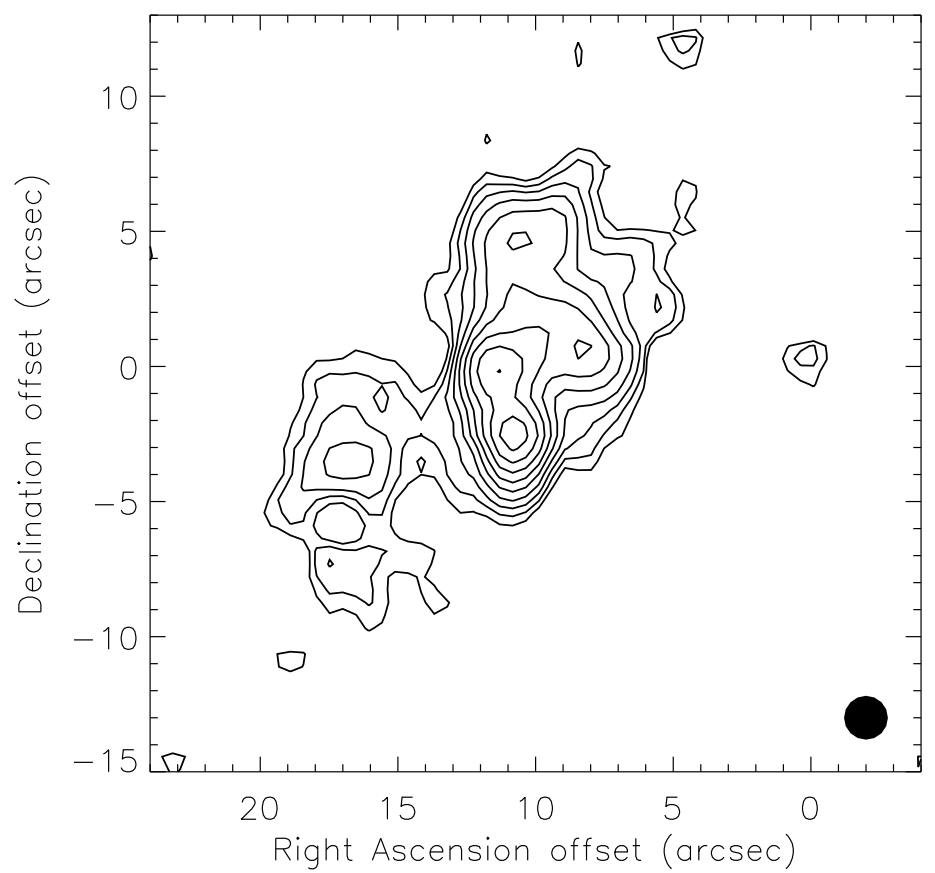

Fig. 6. - A contour plot of the MIRLIN/IRTF $20.8 \mu \mathrm{m}$ image of G11.94-0.62. Same comments as Figure 2a. 


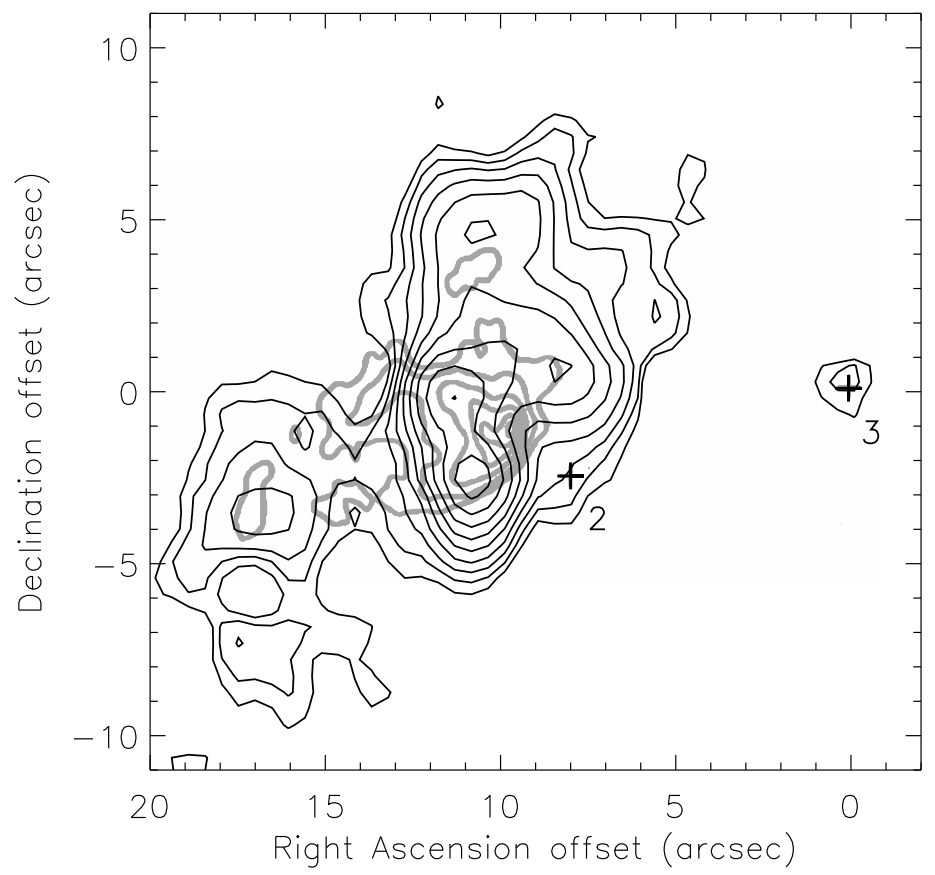

FIG. 7.- A contour plot of the MIRLIN/IRTF $20.8 \mu \mathrm{m}$ image of G11.94-0.62 (black contours) overlaid with the $2 \mathrm{~cm}$ radio continuum image (gray contours) of Hofner \& Churchwell (1996). Also shown are the numbered water maser group locations from Hofner \& Churchwell (1996). The peak of the mid-infrared emission from the HMC candidate is coincident with the location of maser group 3. 


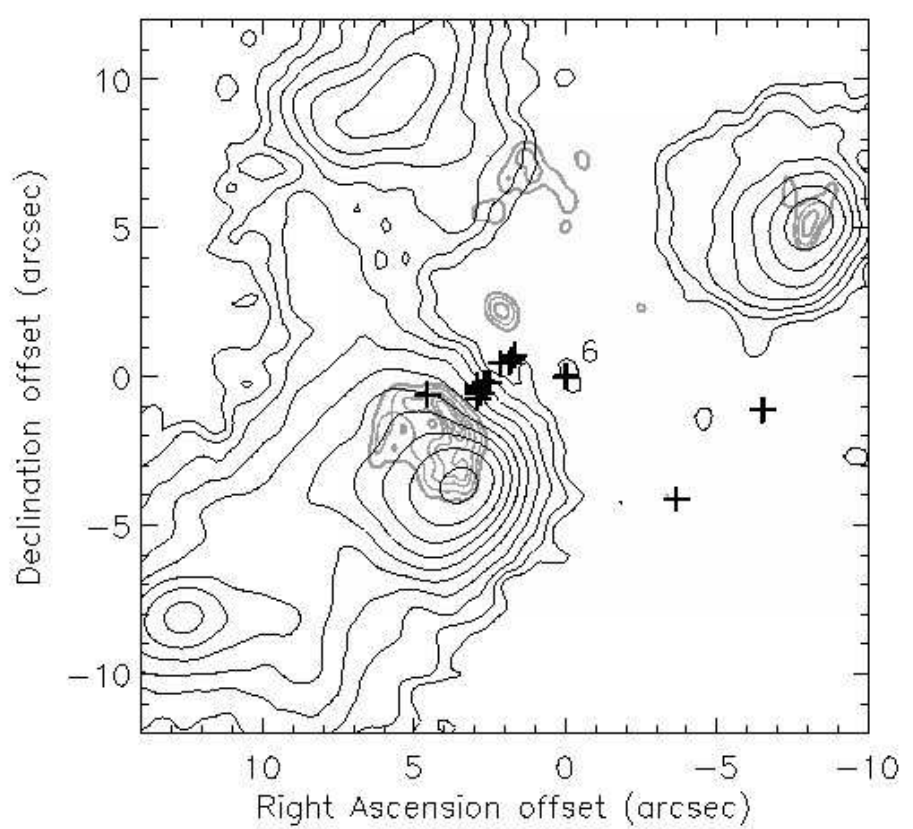

FIG. 8. - A comparison between the $18.1 \mu \mathrm{m}$ image (black contours) of G19.61-0.23 from De Buizer (2000) using OSCIR/IRTF and the $2 \mathrm{~cm}$ radio continuum image (gray contours) from Hofner \& Churchwell (1996). Also shown are the water maser locations from Hofner \& Churchwell (1996), with their water maser group 6 labelled. At this maser group location there is a $3-\sigma$ detection of a mid-infrared source. Given the coincidence of the mid-infrared source with the water maser location and thermal ammonia source of Garay et al. (1998), this mid-infrared detection was considered to be real by De Buizer (2000).

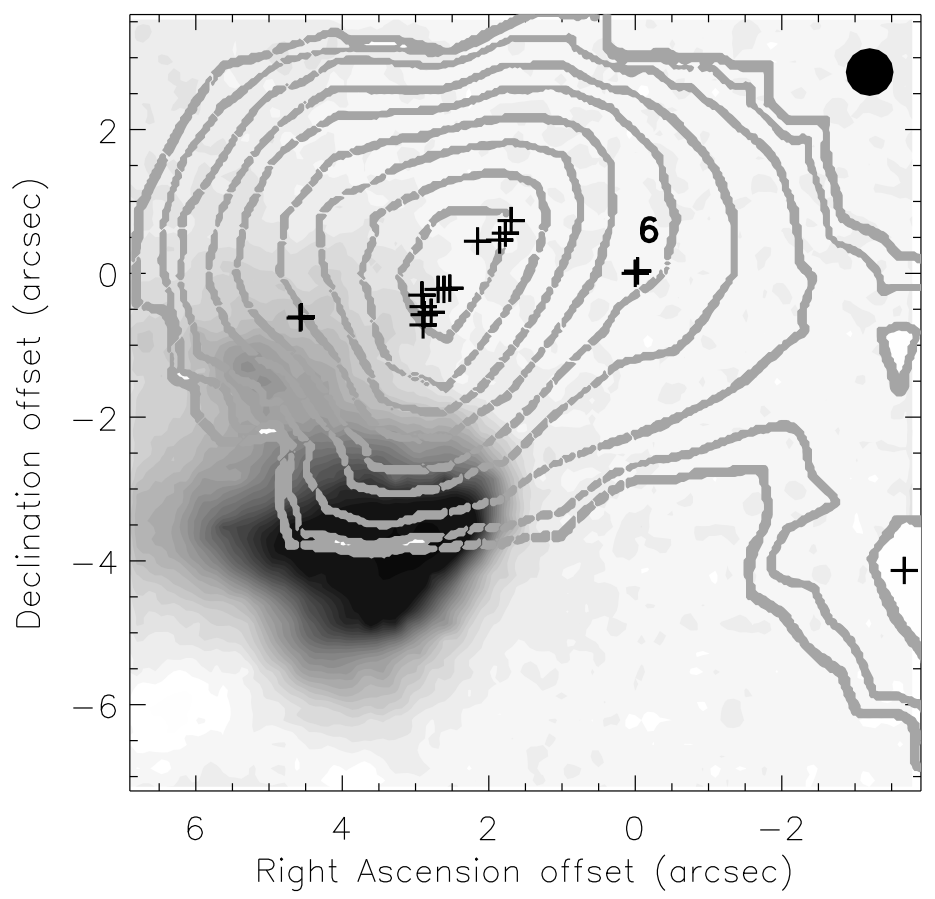

FIG. 9.- Deeper $18.1 \mu \mathrm{m}$ follow-up image of G19.61-0.23 from OSCIR/Gemini North (gray-scale). Overlaid in gray are the contours of the thermal ammonia source seen by Garay et al. (1998). The water masers of Hofner \& Churchwell (1996) are overlaid as well. There was no detection of the $18.1 \mu \mathrm{m}$ source seen by De Buizer (2000) at the location of maser group 6 . Therefore, it is likely that the HMC candidate seen previously was simply noise. The filled circle in the upper right represents the FWHM resolution of the mid-infrared Gemini image. 


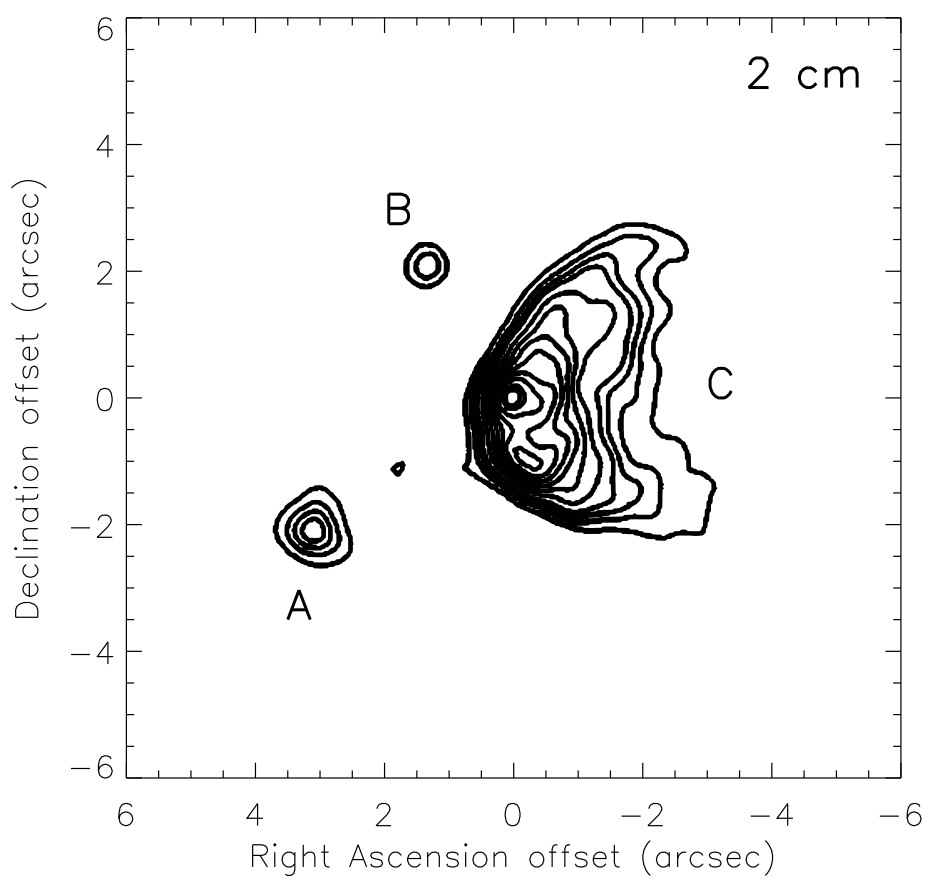

FIG. 10. - A contour plot of the $2 \mathrm{~cm}$ radio continuum of G34.26+0.15 taken from Figure 5 of Hofner \& Churchwell (1996). This figure shows the radio continuum components in the region, which are labelled. The large and amorphous radio source D is not on field and is located $\sim 20^{\prime \prime}$ to the southeast of A. 


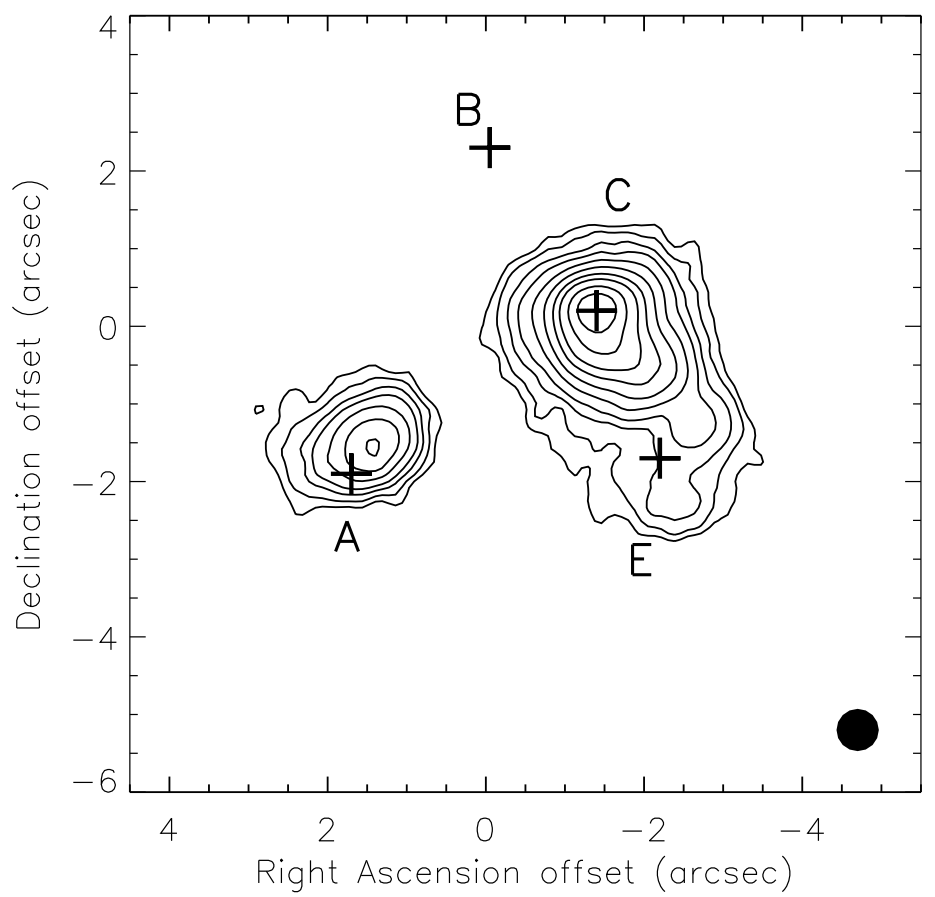

Fig. 11. - A contour plot of the OSCIR/Gemini North $10.5 \mu \mathrm{m}$ image of G34.26+0.15. Labelled crosses A-C mark the peaks of the radio continuum emission sources in the region. Previously detected mid-infrared source E from Campbell et al. (2000) is also marked with a cross. Like G11.94-0.62, the cometary UC HII region, C, in this field does not appear to have a cometary shape in the mid-infrared. It appears that source C and mid-infrared source E (Campbell et al. 2000) can be seen as four separate sources in this high resolution Gemini image. The filled circle in the lower right represents the FWHM resolution of the image.

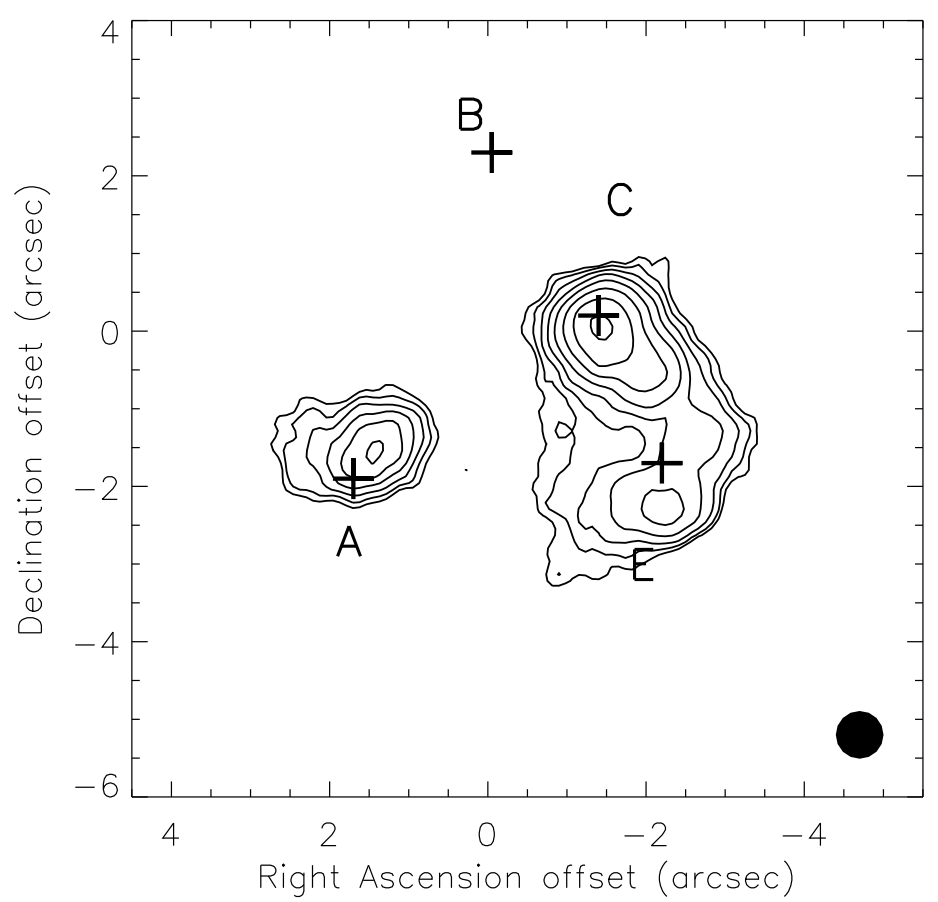

FIG. 12.- The OSCIR/Gemini North $18.1 \mu \mathrm{m}$ image of G34.26+0.15. Same comments as Figure 4a. 


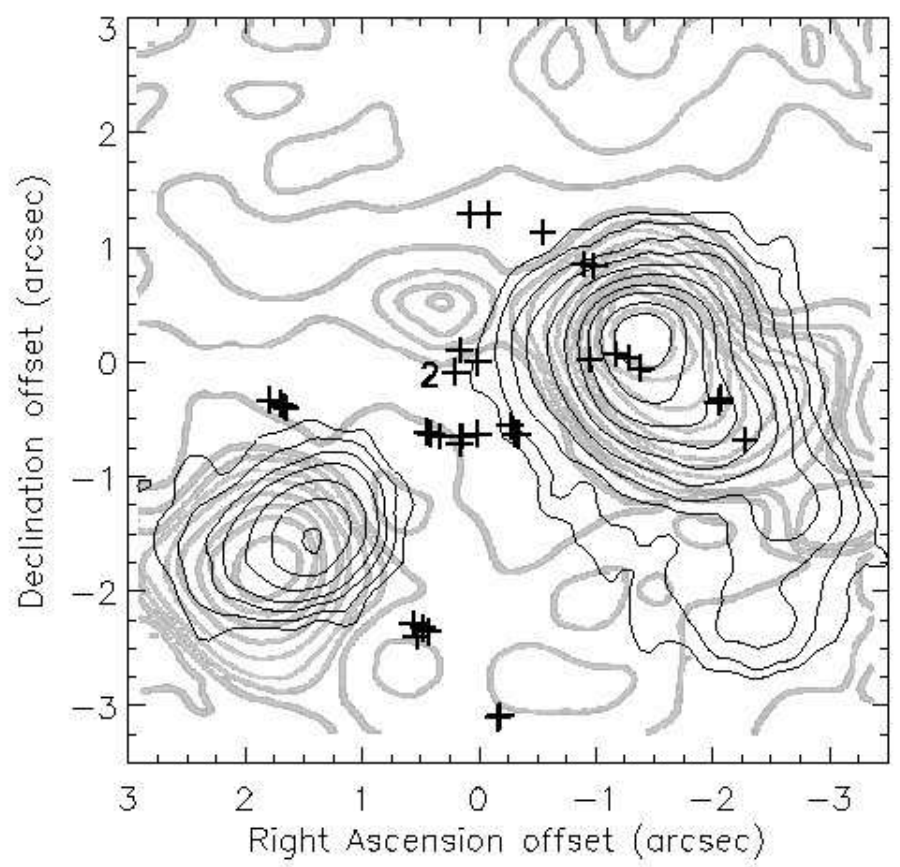

FIG. 13. - The OSCIR/Gemini North $10.5 \mu \mathrm{m}$ image of G34.26+0.15 (black contours) with the $12.5 \mu \mathrm{m}$ image (gray contours) from Keto et al. (1992). Also shown are the water maser group locations from Hofner \& Churchwell (1996). Water maser group 2 is believed to be the site of an HMC. Keto et al. (1992) found a $70 \mathrm{mJy} 12.5 \mu \mathrm{m}$ source near the location of water maser group 2 , claiming it to be mid-infrared emission from the HMC. We do not detect this source at $10.5 \mu \mathrm{m}$ with a $3-\sigma$ upper limit flux density of $4 \mathrm{mJy}$.

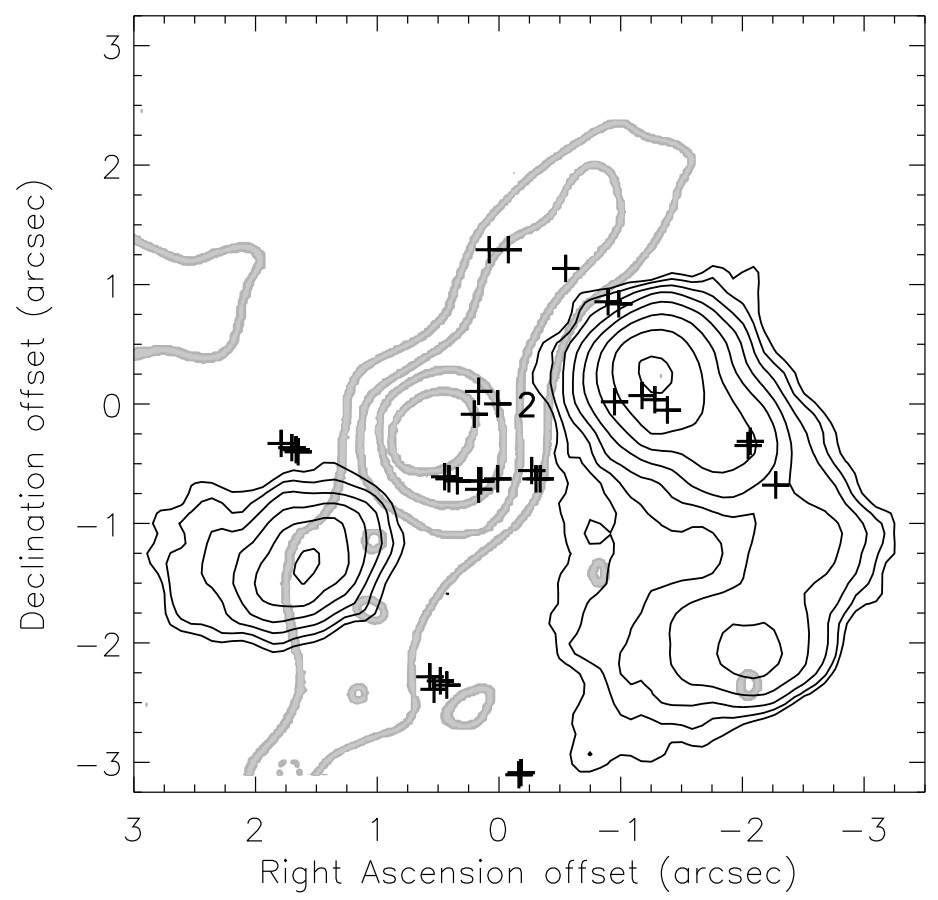

FIG. 14. - The OSCIR/Gemini North $18.1 \mu \mathrm{m}$ image of G34.26+0.15 (black contours) with the thermal $\mathrm{NH}_{3}$ image (gray contours) from Keto et al. (1992). They find a bright thermal ammonia source near the location of water maser group 2. Keto et al. (1992) claim that the water maser group 2, the $12.5 \mu \mathrm{m}$ source, and the ammonia source are spatially coincident, however they appear to be in three different locations. In any case, there is no $18.1 \mu \mathrm{m}$ source at any of these three locations with a $3-\sigma$ upper limit flux density of $21 \mathrm{mJy}$. 


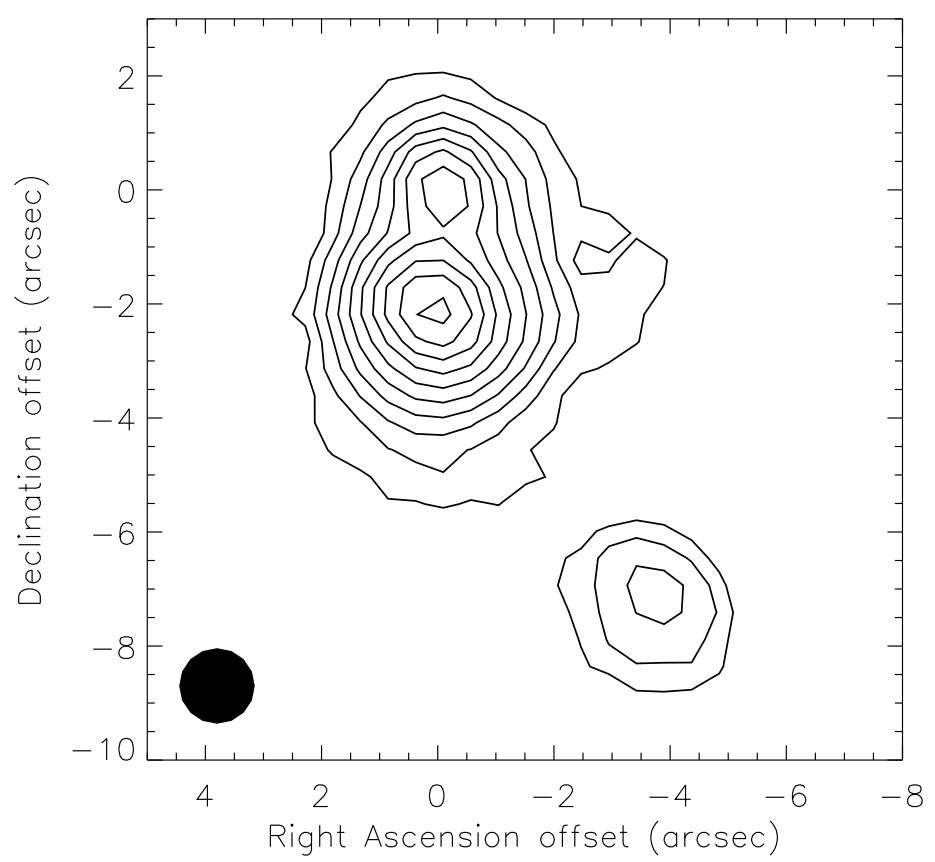

FIG. 15.- A contour plot of the MIRLIN/IRTF $11.7 \mu \mathrm{m}$ image of G45.07-0.13. Three mid-infrared sources were detected, including one at the candidate HMC location (origin). The filled circle in the lower left represents the FWHM resolution of the image.

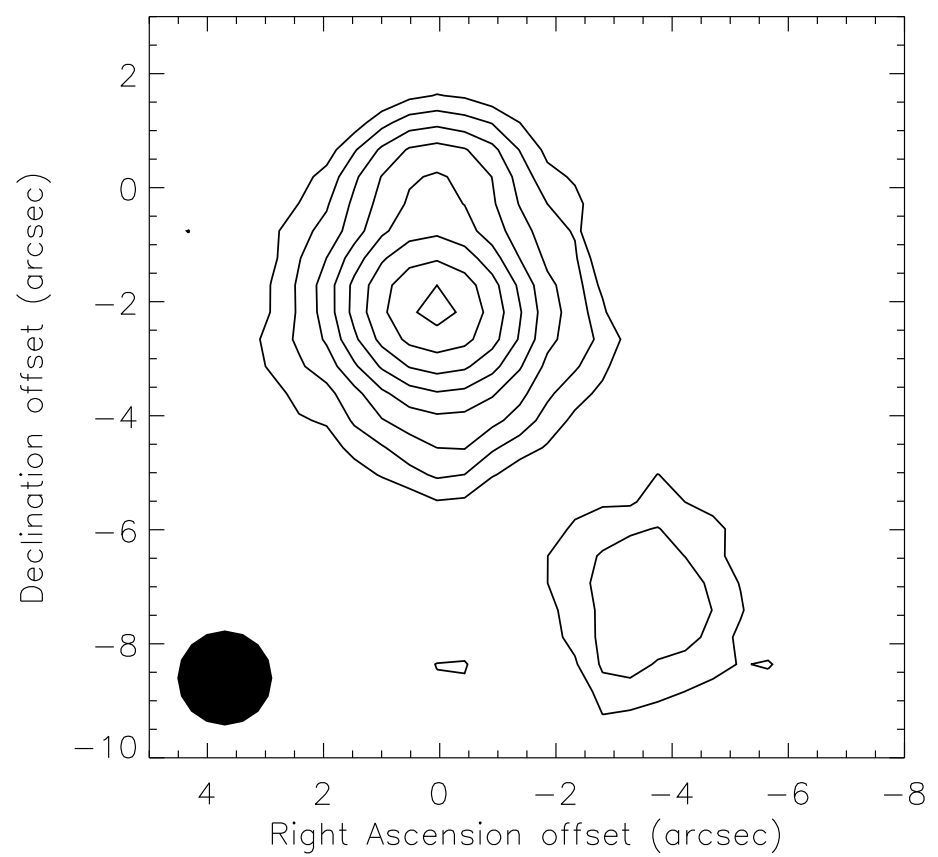

FIG. 16. - A contour plot of the MIRLIN/IRTF $20.8 \mu \mathrm{m}$ image of G45.07-0.13. Again three mid-infrared sources were detected, including one at the candidate HMC location (origin), however the two northern sources are not as spatially well-resolved as they are at $11.7 \mu \mathrm{m}$. The filled circle in the lower left represents the FWHM resolution of the image. 


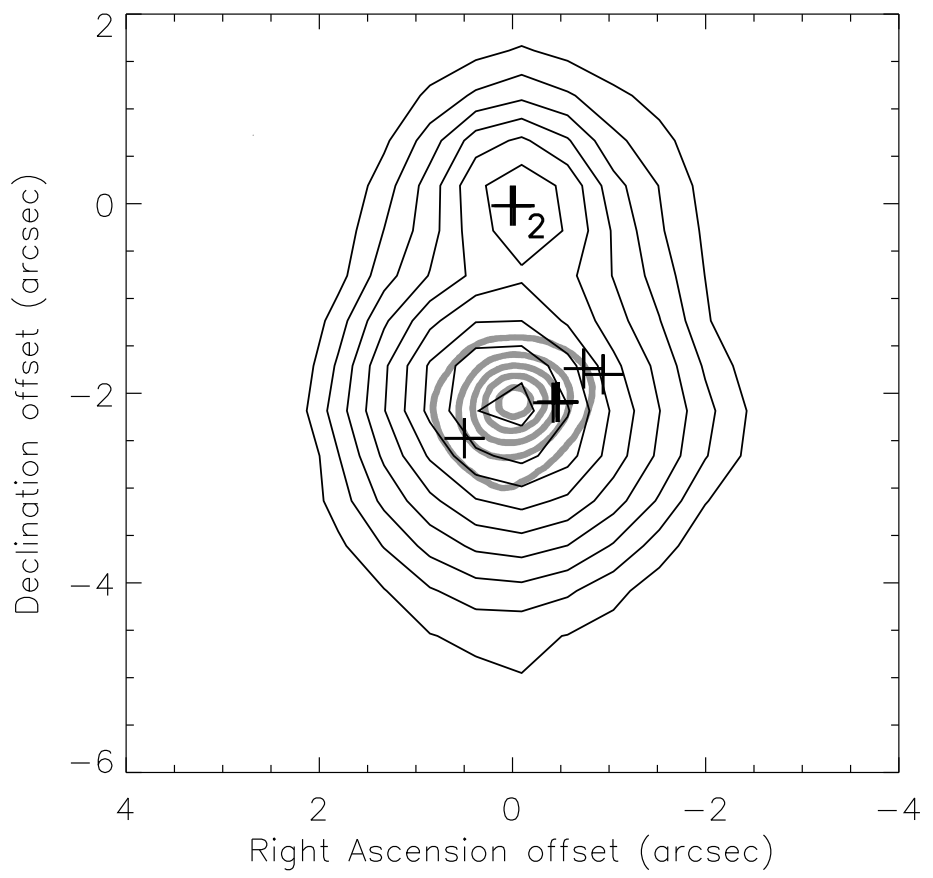

FIG. 17.- The MIRLIN/IRTF $11.7 \mu \mathrm{m}$ image of G45.07-0.13 (black contours) with the $2.0 \mathrm{~cm}$ radio continuum image of Hofner \& Churchwell (1996) overlaid (gray contours). Also shown are the water maser groups of Hofner \& Churchwell (1996). Maser group 2 is believed to mark the location of an HMC, and we have detected a bright mid-infrared source at this location. 